\title{
Characterization of Purple Acid Phosphatase Family and Functional Analysis of GmPAP7a/7b Involved in Extracellular ATP Utilization in Soybean
}

\author{
Shengnan Zhu't, Minhui Chen ${ }^{1 \dagger}$, Cuiyue Liang ${ }^{1}$, Yingbin Xue ${ }^{2}$, Shuling Lin ${ }^{1}$ and \\ Jiang Tian ${ }^{1 *}$
}

1 State Key Laboratory for Conservation and Utilization of Subtropical Agro-Bioresources, Root Biology Center, South China Agricultural University, Guangzhou, China, ${ }^{2}$ Department of Resources and Environmental Sciences, College of Chemistry and Environment, Guangdong Ocean University, Zhanjiang, China

OPEN ACCESS

Edited by: Zhichang Chen,

Fujian Agriculture and Forestry

University, China

Reviewed by:

Weifeng $X u$

Fujian Agriculture and Forestry

University, China

Chuang Wang

Huazhong Agricultural University,

China

*Correspondence:

Jiang Tian

jtian@scau.edu.cn

†These authors have contributed equally to this work

Specialty section: This article was submitted to

Plant Abiotic Stress,

a section of the journal

Frontiers in Plant Science

Received: 17 March 2020

Accepted: 28 April 2020

Published: 24 June 2020

Citation:

Zhu S, Chen M, Liang C, Xue Y,

Lin S and Tian J (2020)

Characterization of Purple Acid

Phosphatase Family and Functional Analysis of GmPAP7a/7b Involved

in Extracellular ATP Utilization

in Soybean. Front. Plant Sci. 11:661.

doi: 10.3389/fpls.2020.00661
Low phosphate (Pi) availability limits crop growth and yield in acid soils. Although root-associated acid phosphatases (APases) play an important role in extracellular organic phosphorus (P) utilization, they remain poorly studied in soybean (Glycine max), an important legume crop. In this study, dynamic changes in intracellular (leaf and root) and root-associated APase activities were investigated under both Pi-sufficient and Pi-deficient conditions. Moreover, genome-wide identification of members of the purple acid phosphatase (PAP) family and their expression patterns in response to $\mathrm{PI}$ starvation were analyzed in soybean. The functions of both GmPAP7a and GmPAP7b, whose expression is up regulated by Pi starvation, were subsequently characterized. Phosphate starvation resulted in significant increases in intracellular APase activities in the leaves after 4 days, and in root intracellular and associated APase activities after 1 day, but constant increases were observed only for root intracellular and associated APase activities during day 5-16 of $\mathrm{P}$ deficiency in soybean. Moreover, a total of 38 GmPAP members were identified in the soybean genome. The transcripts of $19 \mathrm{GmPAP}$ members in the leaves and 17 in the roots were upregulated at 16 days of $P$ deficiency despite the lack of a response for any GmPAP members to Pi starvation at 2 days. $\mathrm{PI}$ starvation upregulated GMPAP7a and GMPAP7b, and they were subsequently selected for further analysis. Both GmPAP7a and GmPAP7b exhibited relatively high activities against adenosine triphosphate (ATP) in vitro. Furthermore, overexpressing GmPAP7a and GmPAP7b in soybean hairy roots significantly increased root-associated APase activities and thus facilitated extracellular ATP utilization. Taken together, these results suggest that GmPAP7a and GmPAP7b might contribute to root-associated APase activities, thus having a function in extracellular ATP utilization in soybean.

Keywords: phosphorus deficiency, soybean, root-associated APase, purple acid phosphatase, ATP utilization

Abbreviations: APase, acid phosphatase; P, phosphorus; PAP, purple acid phosphatase; Pi, phosphate; qRT-PCR, quantitative real-time polymerase chain reaction. 


\section{INTRODUCTION}

Phosphorus (P), an important macronutrient, is involved in many biochemical and metabolic processes in plants, such as photosynthesis, nucleotide synthesis, membrane remodeling, and protein modification (Liang et al., 2010; Zhang et al., 2014; Ham et al., 2018). However, a large proportion of $\mathrm{P}$ exists in immobile forms (i.e., organic P esters and inorganic complexes) and is thus unavailable for plant utilization in most soils, especially in acid soils (Chiou and Lin, 2011; Tian et al., 2012b; Plaxton and Lambers, 2015). Therefore, Pi fertilizer application is required to meet the $\mathrm{Pi}$ requirements for crop production. However, excess application of $\mathrm{Pi}$ fertilizer causes serious environmental eutrophication (Conley et al., 2009; Veneklaas et al., 2012; Abel, 2017; Wang and Liu, 2018). Thus, breeding cultivars with high $\mathrm{P}$ efficiency and optimizing field $\mathrm{P}$ management practices are necessary to maintain sustainable agricultural development (Tian et al., 2012b; Abel, 2017). It has been well documented that plants have evolved complex adaptation strategies to increase Pi foraging and recycling, such as altering root morphology and architecture, increasing organic acid and PAP exudation, and enhancing root-microbe interactions (Chiou and Lin, 2011; Liang et al., 2014; Ham et al., 2018; Jung et al., 2018).

In terms of adaptive strategies, plant PAPs are believed to play a vital role in Pi mobilization (Tran et al., 2010a; Plaxton and Lambers, 2015; Tian and Liao, 2015; Wang and Liu, 2018). It is generally observed that plant PAPs harbor a binuclear metal center binding either Fe (III)-Zn (II) or Fe (III)-Mn (II) ions and comprise five conserved motif blocks (DXG/GDXXY/GNH(D/E)/VXXH/GHXH) (Tran et al., 2010a; Tian and Liao, 2015). With the aid of genome sequence availability for plant species, a variety of PAPs have been identified and annotated. For example, there are 29 annotated PAP members in Arabidopsis (Arabidopsis thaliana), 26 in rice (Oryza sativa), 33 in maize (Zea mays), 25 in chickpea (Cicer arietinum), and 25 in physic nut (Jatropha curcas) (Li et al., 2002; Zhang et al., 2010; Gonzalez-Munoz et al., 2015; Bhadouria et al., 2017; Venkidasamy et al., 2019). Plant PAPs are widely divided into two major groups on the basis of PAP mass and structure: low molecular-mass monomeric PAPs, with a mass of approximately $35 \mathrm{kD}$, and high molecular-mass oligomeric PAPs, with a subunit mass of approximately $55 \mathrm{kD}$ (Olczak et al., 2003; Tran et al., 2010a; Plaxton and Lambers, 2015; Tian and Liao, 2015).

It has been documented that $\mathrm{P}$ deficiency enhances the expression levels of most plant PAPs. For example, in rice, 10 out of 26 PAP members (OsPAP1a, 1d, 3b, 9b, 10a, 10c, 20b, $21 b, 23$, and $27 a$ ) are upregulated in the roots under P-deficient conditions (Zhang et al., 2010). Similarly, Pi starvation enhanced transcripts of PAP members are also observed in 11 of 33 PAP members in maize, at least 20 of 25 PAP members in physic nut, 11 of 29 PAP members in Arabidopsis, 23 of 35 PAP members in soybean (Glycine max), and 12 of 25 PAP members in chickpea (Del Pozo et al., 1999; Haran et al., 2000; Li C. C. et al., 2012; Wang et al., 2014; Gonzalez-Munoz et al., 2015; Bhadouria et al., 2017; Venkidasamy et al., 2019). Therefore, it is suggested that increased transcription levels of PAPs could contribute to significant increases in intracellular and extracellular APase activities in plant species, such as Arabidopsis, wheat (Triticum aestivum), soybean, and tomato (Lycopersicon esculentum) (Del Pozo et al., 1999; Li et al., 2002; Kaida et al., 2003; Zimmermann et al., 2004; Liang et al., 2010; Chiou and Lin, 2011).

Recently, accumulating evidence has suggested that most PAP members participate in extracellular organic $\mathrm{P}$ utilization, including adenosine triphosphate (ATP), deoxynucleotide triphosphate (dNTPs), and phytate-P (Tran et al., 2010a; Plaxton and Lambers, 2015; Tian and Liao, 2015; Wang and Liu, 2018). A pioneer study on PAP function in extracellular ATP utilization has been conducted in common bean (Phaseolus vulgaris) in which overexpression of Pi starvation-enhanced $P v P A P 3$ resulted in significant increases of fresh weight and $\mathrm{P}$ content in bean hairy roots cultured in media supplemented with ATP as the sole $P$ source (Liang et al., 2010). Similar functions have also been observed for other PAP members, such as OsPAP10a, OsPAP21b, OsPAP26, and OsPAP10c in rice (Tian et al., 2012a; Lu et al., 2016; Gao et al., 2017; Mehra et al., 2017). In addition to extracellular ATP utilization, plant PAP members have also been suggested to participate in extracellular dNTP utilization, including $P v P A P 1 / 3$ from common bean, SgPAP7/10/26 from stylo (Stylosanthes spp.) and GmPAP1-like from soybean (Liang et al., 2012; Liu et al., 2016; Wu et al., 2018). Recently, some PAP members exhibiting phytase activity have been suggested to mediate extracellular phytate utilization, including SgPAP23 from stylo, OsPHY1 from rice, MtPHY1 from Medicago (Medicago truncatula), and GmPAP4 and GmPAP14 from soybean (Ma et al., 2009; Li R. J. et al., 2012; Kong et al., 2014, 2018; Liu et al., 2018), suggesting diverse functions of PAP members in $\mathrm{P}$ scavenging and recycling in plants.

Soybean is an important legume crop species and an important oil and high-protein food or forage crop species worldwide (Conner et al., 2004; Herridge et al., 2008). In the face of low Pi availability conditions, soybean has evolved strategies to maintain $\mathrm{Pi}$ homeostasis, including the formation of a shallower root system, increased organic acid exudation and APase activities, and the formation of symbiotic associations with arbuscular mycorrhizal (AM) fungi (Tian et al., 2003; Zhao et al., 2004; Liao et al., 2006; Liu et al., 2008; Wang et al., 2010; Li et al., 2019). Furthermore, a functional analysis of several Pi starvation-responsive genes, such as GmEXPB2, GmPHR25, GmPT5/7, and GmSPX1/3, has highlighted molecular mechanisms underlying soybean adaptation to Pi starvation (Li et al., 2014, 2015; Yao Z. et al., 2014; Xue et al., 2017; Chen et al., 2018). However, the dynamic changes in intracellular and root-associated APase activities in soybean in response to $\mathrm{Pi}$ starvation, and the functions of GmPAP in extracellular ATP utilization remain unclear. Therefore, in this study, dynamic changes of intracellular (leaves and roots) and root-associated APase activities were investigated under both Pi-sufficient and Pi-deficient conditions. Moreover, genome-wide identification of members of the PAP family, and their expression patterns in response to Pi starvation were analyzed in soybean. Furthermore, the functions of the Pi starvation responsive GmPAP7a and $G m P A P 7 b$ genes are suggested to participate in extracellular ATP utilization in soybean. 


\section{MATERIALS AND METHODS}

\section{Plant Materials and Growth Conditions}

The soybean genotype YC03-3 was used in this study. To analyze dynamic changes in APase activities at two P levels, soybean seeds were surface-sterilized and rolled in absorbent papers, which are soaked with one-fourth strength nutrient solution as described previously (Liang et al., 2010). After 5 days of germination, uniform seedlings were transferred to a nutrient solution comprising the following components (in $\mu \mathrm{M}): 1500 \mathrm{KNO}_{3}, 1200 \mathrm{Ca}\left(\mathrm{NO}_{3}\right)_{2}, 400 \mathrm{NH}_{4} \mathrm{NO}_{3}, 25 \mathrm{MgCl}_{2}$, $500 \mathrm{MgSO}_{4}, \quad 300 \mathrm{~K}_{2} \mathrm{SO}_{4}, 0.3\left(\mathrm{NH}_{4}\right)_{2} \mathrm{SO}_{4}, \quad 1.5 \mathrm{MnSO}_{4}, \quad 0.5$ $\mathrm{CuSO}_{4}, 1.5 \mathrm{ZnSO}_{4}, 0.16\left(\mathrm{NH}_{4}\right)_{6} \mathrm{Mo}_{7} \mathrm{O}_{24}, 2.5 \mathrm{NaB}_{4} \mathrm{O}_{7}, 40 \mathrm{Fe}-$ $\mathrm{Na}-E D T A$, and $5(-\mathrm{P})$ or $250(+\mathrm{P}) \mathrm{KH}_{2} \mathrm{PO}_{4}$, as previously described (Wu et al., 2018). The nutrient solution was aerated hourly and refreshed weekly. Moreover, the $\mathrm{pH}$ value of the nutrient solution was adjusted to 5.8 every 2 days. The fresh weight of the soybean shoots and roots was determined daily within 7 days after $\mathrm{P}$ treatments were applied, and at 10, 13, and 16 days after $\mathrm{P}$ treatments were applied. Moreover, the roots were harvested to determine total $\mathrm{P}$ concentration, intracellular and root-associated APase activities. Primary leaves were also harvested to determine total $\mathrm{P}$ concentration and intracellular APase activities except at 0 and after 1 days of $\mathrm{P}$ treatment. To assay the temporal expression patterns of GmPAP members in response to $\mathrm{Pi}$ starvation, the primary leaves and roots after 2 days and 16 days of $\mathrm{P}$ treatments were also separately harvested for further analysis. All experiments had four biological replicates.

\section{Total P Concentration and APase Activity Analysis}

The total $\mathrm{P}$ concentration was measured as described previously (Murphy and Riley, 1963; Xue et al., 2017; Mo et al., 2019). Briefly, approximately $0.1 \mathrm{~g}$ of dry samples was ground and digested using $\mathrm{H}_{2} \mathrm{SO}_{4}-\mathrm{H}_{2} \mathrm{O}_{2}$ reagent. Afterward, the mixtures were reacted with ammonium molybdate reagent and measured $30 \mathrm{~min}$ later at $700 \mathrm{~nm}$.

To determine intracellular APase activities, the reaction product hydrolyzed by APase, $\rho$-nitrophenol ( $\rho$-NP), was measured at an absorbance of $405 \mathrm{~nm}$ as described previously (Liang et al., 2010; Liu et al., 2016; Wu et al., 2018). Briefly, approximately $0.1 \mathrm{~g}$ of leaves and roots was ground and homogenized with $1.2 \mathrm{~mL} 0.1 \mathrm{M}$ Tris- $\mathrm{HCl}(\mathrm{pH}$ 8.0), and then the mixtures were centrifuged at $12,000 \mathrm{~g}$ for $30 \mathrm{~min}$. After centrifugation, the supernatants were mixed with $1.8 \mathrm{~mL}$ of $45 \mathrm{mM}$ Na-acetate buffer ( $\mathrm{pH} 5.0$ ) consisting of $1 \mathrm{mM} \rho$-nitrophenyl phosphate $(\rho$-NPP) as the substrate at $37^{\circ} \mathrm{C}$ for $15 \mathrm{~min}$. After adding $1 \mathrm{M} \mathrm{NaOH}$, the released $\rho$-NP was measured spectrophotometrically at $405 \mathrm{~nm}$. The protein content in the supernatants was quantified using the Coomassie brilliant blue method (Bradford, 1976). Intracellular APase activities were expressed as the amount of $\rho$-NP produced per minute per milligram protein.
To analyze root-associated APase activities, seedlings grown in a nutrient solution containing $250 \mu \mathrm{M}(+\mathrm{P})$ or $5 \mu \mathrm{M}(-\mathrm{P})$ $\mathrm{KH}_{2} \mathrm{PO}_{4}$ were rinsed in a $0.2 \mathrm{M} \mathrm{CaCl} 2$ solution once and deionized water three times. After that, the whole roots of seedlings subjected to 0-7 days of $\mathrm{P}$ treatments and detached partial lateral roots of seedlings at 10-16 days of $\mathrm{P}$ treatment were transferred separately into tubes containing $40 \mathrm{~mL}$ of reaction buffer containing $45 \mathrm{mM}$ Na-acetate buffer ( $\mathrm{pH} 5.0$ ) and $1 \mathrm{mM} \rho$-NPP. The reactions were incubated at $25^{\circ} \mathrm{C}$ for $2 \mathrm{~h}$ to allow the reactions to occur, and they were then terminated by the addition of $1 \mathrm{M} \mathrm{NaOH}$. The absorbance of the reaction mixtures was separately measured at $405 \mathrm{~nm}$ (Liu et al., 2016). Moreover, the fresh weight of the roots was also determined. Root-associated APase activities were calculated as the amount of released $\rho$-NP per minute per gram of fresh roots.

To visualize root-associated APase activities, the roots were incubated in solid Murashige and Skoog (MS) media containing the substrate 5-bromo-4-chloro-3-indolyl-phosphate (BCIP) (Sigma-Aldrich, United States) as described previously (Wang et al., 2014). Briefly, the detached roots were placed on solid MS media containing $0.03 \%$ agar and then covered with solid MS media containing $0.06 \%$ agar and $0.1 \%(\mathrm{w} / \mathrm{v})$ BCIP. After incubation at $28^{\circ} \mathrm{C}$ for $2 \mathrm{~h}$, the cleavages of BCIP showed a bright blue color, which was imaged by a single-lens reflex camera (Canon, Japan). Each experiment was conducted for at least four biological replicates.

\section{Bioinformatic and Phylogenetic Tree Analysis of PAPs}

For bioinformatic analysis, the sequences of PAP members from soybean were extracted from the $\mathrm{NCBI}^{1}$ and Phytozome ${ }^{2}$ databases. After multiple sequence alignment using ClustalX and conserved domain (metallophos domain) searches using the SMART tool ${ }^{3}$ (Bhadouria et al., 2017), GmPAP members were identified and named on the basis of their homology with corresponding PAP members in Arabidopsis. Detailed information about the GmPAP members, including their protein mass, number of amino acids, subcellular localization, and $\mathrm{N}$-glycosylation site prediction, was retrieved separately from servers, including the ExPASy ${ }^{4}$, TargetP $1.1^{5}$ and NetNGlyc 1.0 server $^{6}$ as described previously (Bhadouria et al., 2017; Xue et al., 2017; Yin et al., 2019). Moreover, a group of PAP members with known functions from other species, such as common bean, rice, maize, Arabidopsis, lupinus (Lupinus luteus), sweet potato (Ipomoea batatas), wheat, tobacco (Nicotiana tabacum), barely (Hordeum vulgare), stylo, Medicago, white lupin (Lupinus albus), potato (Solanum tuberosum), and chickpea, were retrieved from the NCBI database (Kaida et al., 2003; Li and Wang, 2003; Ma et al., 2009; Dionisio et al., 2011; Li C. C. et al., 2012;

\footnotetext{
${ }^{1}$ https://www.ncbi.nlm.nih.gov/pubmed

${ }^{2}$ https://phytozome.jgi.doe.gov/pz/portal.html

${ }^{3}$ http://smart.embl-heidelberg.de/smart/set_mode.cgi?NORMAL=1

${ }^{4}$ https://web.expasy.org/protparam/

${ }^{5}$ http://www.cbs.dtu.dk/services/TargetP-1.1/index.php

${ }^{6}$ http://www.cbs.dtu.dk/services/NetNGlyc/
} 

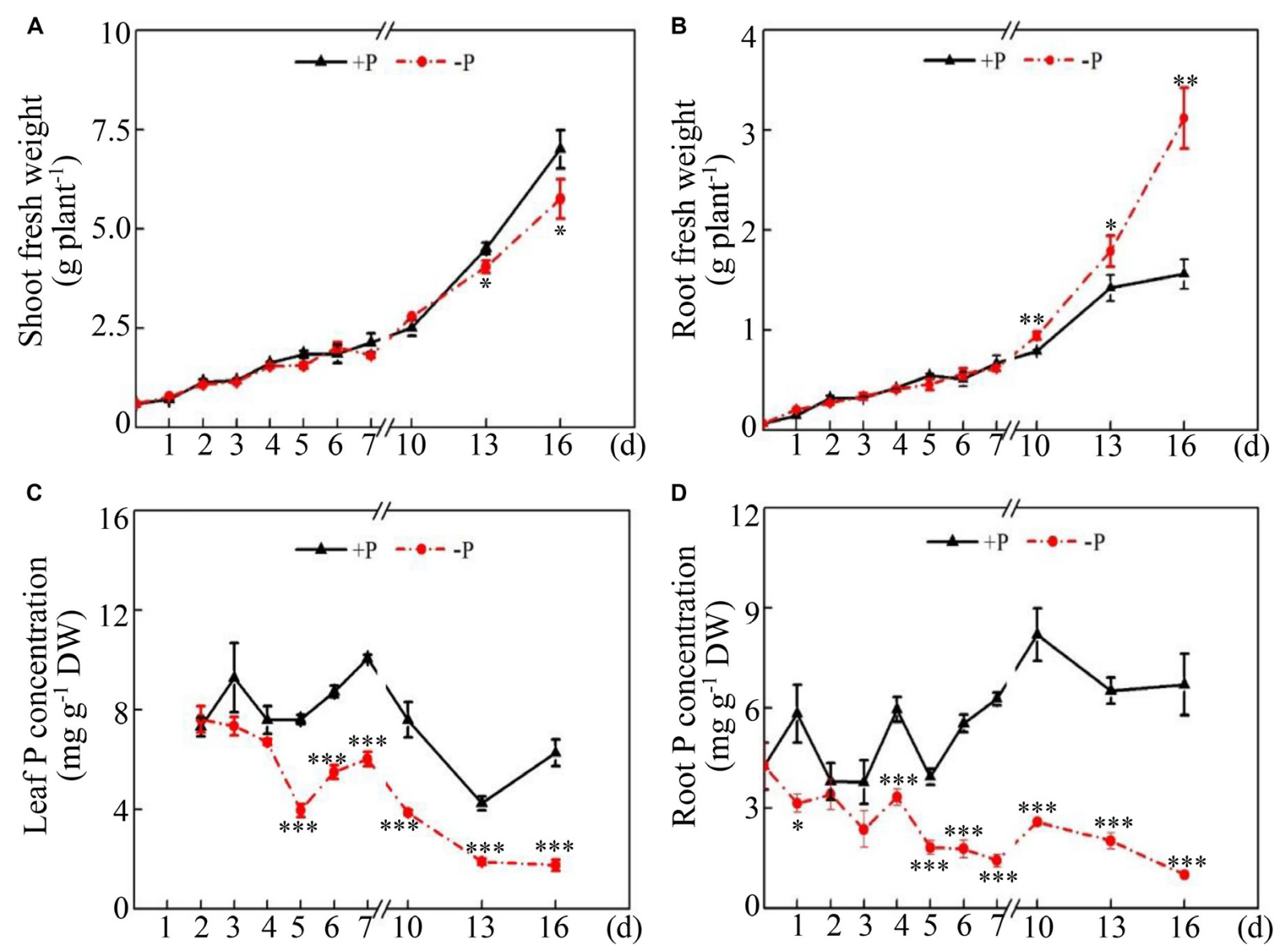

FIGURE 1 | Dynamic changes in soybean growth and P concentration at two P levels. (A) Shoot fresh weight. (B) Root fresh weight. (C) Leaf P concentration. (D) Root $\mathrm{P}$ concentration. Soybean seedlings were grown in nutrient solution supplemented with $5 \mu \mathrm{M}(-\mathrm{P})$ or $250 \mu \mathrm{M}(+\mathrm{P}) \mathrm{KH}_{2} \mathrm{PO}$. Fresh weight and $\mathrm{P}$ concentration were dynamically measured. The data are the means of four replicates with standard errors. The asterisks indicate significant differences between the $-\mathrm{P}$ and $+\mathrm{P}$ treatments according to Student's $t$-test: ${ }^{*} P<0.05 ;{ }^{* *} 0.001<P<0.01 ;{ }^{* * *} P<0.001$.

Madsen et al., 2013; Bhadouria et al., 2017; Liu et al., 2018). A phylogenetic tree was constructed using MEGA 5.01 with 1000 bootstrap values using the neighbor-joining method as described previously (Xue et al., 2017).

\section{RNA Extraction and Quantitative RT-PCR Analysis}

Total RNA was extracted and purified as described previously (Xue et al., 2018). To eliminate genomic DNA contamination, the total RNA was further treated with RNase-free DNase I (Invitrogen, United States), and the purity was evaluated via A260/A280 ratios by using a Nanodrop spectrophotometer (Thermo, United States). Approximately $1 \mu \mathrm{g}$ of RNA was reversely transcribed using MMLV-reverse transcriptase (Promega, United States) following the manufacturer's protocols. Synthesized first-strand cDNA was used for SYBR Greenmonitored quantitative RT-PCR (qRT-PCR) analysis on a Rotor-Gene 3,000 real-time PCR system (Corbett Research, Australia). The primers used for GmPAP members and the housekeeping gene GmEF1- $\alpha$ (Glyma.17G186600) are detailed in the Supplementary Table S1. Relative transcript levels of soybean GmPAP members were also presented using a heatmap generated by TBtools software.

\section{Subcellular Localization and Histochemical Localization of GmPAP7a/7b}

The full-length GmPAP7a/7b coding sequence without a stop codon was separately amplified using the gene specific primers GmPAP7a/7b-GFP-F/R (Supplementary Table S1) and then cloned into a $P E G A D$ vector to generate 35S:GmPAP7a/7bGFP fusion constructs. The constructs fusion with GFP and the plasma membrane marker AtPIP2A-mCherry were co-transformed into tobacco epidermal cells as described previously (Liu et al., 2016). The fluorescent signals were observed via a Zeiss LSM7 Duo confocal microscope (Zeiss, Germany) at $488 \mathrm{~nm}$ for GFP and $568 \mathrm{~nm}$ for mCherry (Liu et al., 2018). Fluorescent images were further processed using Zen2011 software (Carl Zeiss Microscopy, Germany). A $3.0 \mathrm{~kb}$ upstream sequence of GmPAP7a/7b from the start 

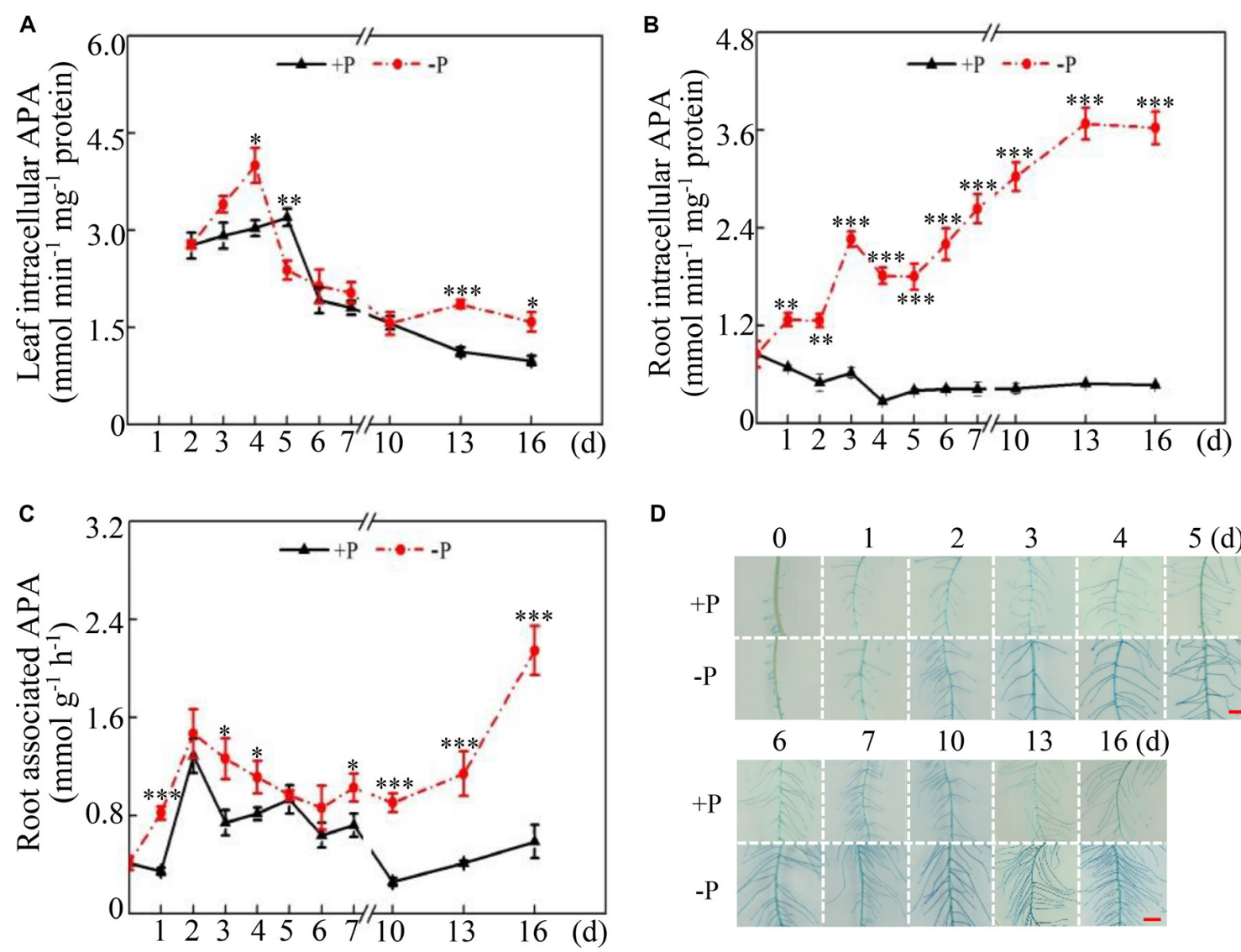

D

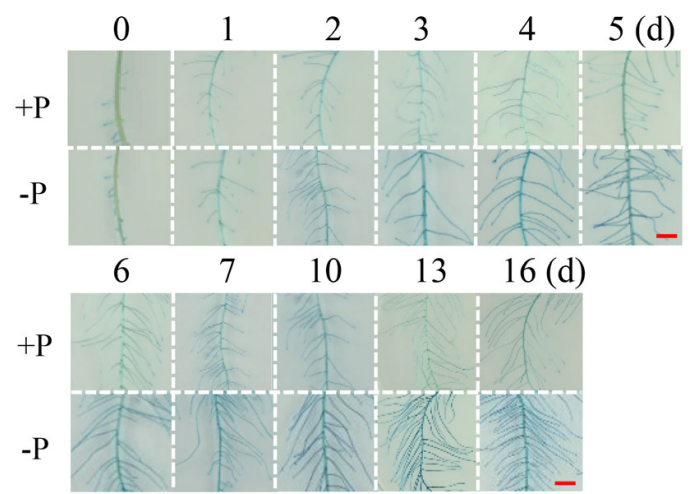

FIGURE 2 | Dynamic changes in intracellular and root-associated APase activities in soybean. (A) Leaf intracellular APase activities. (B) Root intracellular APase activities. (C) Root-associated APase activities. (D) Root-associated APase activities detected by BCIP staining. Soybean seedlings were grown in nutrient solution supplemented with $5 \mu \mathrm{M}(-\mathrm{P})$ or $250 \mu \mathrm{M}(+\mathrm{P}) \mathrm{KH}_{2} \mathrm{PO}_{4}$. Intracellular and associated APase activities were dynamically measured. Data are means of four replicates with standard errors. Asterisks indicate significant differences between the $-\mathrm{P}$ and $+\mathrm{P}$ treatments by according to Student's $t$-test: ${ }^{*} P<0.05$; ${ }^{* *} 0.001<P<0.01$; *** $P<0.001$. The bars $=1 \mathrm{~cm}$.

of codon was amplified using primers GmPAP7a/7b-GUS-F/R (Supplementary Table S1) and then inserted into a pTF102 plasmid containing $\beta$-glucuronidase (GUS). The plasmids were subsequently transformed into soybean hairy roots for analysis of their histochemical localization. Transgenic soybean hairy roots were cultivated in MS media supplied with $1250 \mu \mathrm{M}(+\mathrm{P})$ or $5 \mu \mathrm{M} \mathrm{KH} \mathrm{KO}_{4}(-\mathrm{P})$ for 1 week and then incubated in GUS staining solution $\left(0.1 \mathrm{M} \mathrm{Na} \mathrm{HPO}_{4} / \mathrm{NaH}_{2} \mathrm{PO}_{4}\right.$ buffer, $\mathrm{pH}$ 7.0, $1 \mathrm{mM} \mathrm{X}$-Gluc) for $8 \mathrm{~h}$ at $37^{\circ} \mathrm{C}$ as described previously (Jefferson et al., 1987). After GUS staining, the root samples were separately observed under a light microscope (Leica, Germany).

\section{Purification and Biochemical Characterization of GmPAP7a/7b}

The entire coding sequences of both GmPAP7a and GmPAP7b were separately cloned into a $p G E X-6 P-3$ vector (GE Healthcare, United States) constructs fusion to a GST tag using an in-fusion cloning kit (TAKARA, United States) with primers GmPAP7a/7bGST-F/R (Supplementary Table S1). The expression constructs were introduced into Escherichia coli (E. coli) strain BL21.
Recombinant proteins were extracted using BeaverBeads ${ }^{\mathrm{TM}}$ GSH magnetic beads (Beaver Nano, China) and recognized by immunoblot analysis using anti-GST antibodies as described previously (Liu et al., 2018). Purified proteins were used to analyze their enzymatic properties. The effects of different $\mathrm{pH}$ values (ranging from 3.0 to 9.0) on GmPAP7a/7b activities were estimated separately by the addition of $5 \mathrm{mM} \rho$-NPP to the reaction buffers for $15 \mathrm{~min}$ at $37^{\circ} \mathrm{C}$ as described previously (Liu et al., 2018). The reaction buffer included $45 \mathrm{mM}$ of glycine- $\mathrm{HCl}$ buffer ( $\mathrm{pH} 3.0-4.5)$, Na-acetate buffer ( $\mathrm{pH} 5.0-5.5)$, Tris- $\mathrm{HCl}$ 2-(N-morpholino)-ethanesulfonic acid (MES) buffer ( $\mathrm{pH}$ 6.07.0), and Tris-HCl buffer ( $\mathrm{pH} 7.5-9.0$ ). Moreover, the effects of different temperatures (over a range of $20-80^{\circ} \mathrm{C}$ ) on the enzymatic activities were also measured in $45 \mathrm{mM}$ Na-acetate buffer ( $\mathrm{pH} 5.0$ ) with $5 \mathrm{mM} \rho$-NPP used as a substrate. The relative activities of GmPAP7a/7b were calculated as the percentages of their activities out of the highest activities under different $\mathrm{pH}$ values or temperatures. Moreover, a broad range of substrates were added to $45 \mathrm{mM} \mathrm{Na}$-acetate buffer ( $\mathrm{pH} \mathrm{5.0)}$ ) to test their substrate specificities, including the following (at concentrations of $5 \mathrm{mM}$ ): phytate-P, ATP, adenosine diphosphate (ADP), 
TABLE 1 | General information of GmPAP members in soybean.

\begin{tabular}{|c|c|c|c|c|c|c|c|}
\hline Name & Locus & $\begin{array}{l}\text { Chromosomal } \\
\text { location }\end{array}$ & $\begin{array}{l}\text { Length of } \\
\text { ORF (bp) }\end{array}$ & $\begin{array}{c}\text { Number of amino } \\
\text { acids (aa) }\end{array}$ & $\begin{array}{l}\text { Protein size } \\
\text { (kD) }\end{array}$ & $\begin{array}{l}\text { Subcellular } \\
\text { location }\end{array}$ & $\begin{array}{c}\mathrm{N} \text {-glycosylation } \\
\text { sites }\end{array}$ \\
\hline GmPAP1a & Glyma.02G117000.1 & 2 & 1926 & 642 & 72 & - & 5 \\
\hline GmPAP18a & Glyma.02G290100.1 & 2 & 1365 & 454 & 51 & $S$ & 1 \\
\hline GmPAP22a & Glyma.03G194100.1 & 3 & 1476 & 491 & 55 & C & 2 \\
\hline GmPAP20a & Glyma.03G194200.1 & 3 & 1293 & 432 & 48 & S & 3 \\
\hline GmPAP27c & Glyma.04G195600.1 & 4 & 1560 & 519 & 59 & M & 0 \\
\hline GmPAP27a & Glyma.05G047900.1 & 5 & 1875 & 524 & 70 & $S$ & 5 \\
\hline GmPAP7d & Glyma.05G138300.1 & 5 & 933 & 310 & 35 & S & 0 \\
\hline GmPAP7a & Glyma.05G138400.1 & 5 & 1008 & 335 & 37 & S & 2 \\
\hline GmPAP17d & Glyma.05G247800.1 & 5 & 1065 & 354 & 40 & S & 1 \\
\hline GmPAP17a & Glyma.05G247900.1 & 5 & 996 & 331 & 37 & $S$ & 1 \\
\hline GmPAP12a & Glyma.06G028100.1 & 6 & 1135 & 444 & 51 & - & 5 \\
\hline GmPAP12b & Glyma.06G028200.1 & 6 & 1425 & 474 & 54 & $S$ & 4 \\
\hline GmPAP27b & Glyma.06G170300.1 & 6 & 1872 & 623 & 70 & $S$ & 5 \\
\hline GmPAP17b & Glyma.08G056400.1 & 8 & 987 & 328 & 37 & $S$ & 1 \\
\hline GmPAP7e & Glyma.08G093300.1 & 8 & 969 & 322 & 36 & S & 2 \\
\hline GmPAP7c & Glyma.08G093500.1 & 8 & 999 & 332 & 38 & S & 1 \\
\hline GmPAP7b & Glyma.08G093600.1 & 8 & 1008 & 335 & 38 & S & 1 \\
\hline GmPAP1b & Glyma.08G291600.1 & 8 & 1856 & 616 & 69 & S & 3 \\
\hline GmPAP18b & Glyma.08G314800.1 & 8 & 1410 & 469 & 53 & - & 1 \\
\hline GmPAP15a & Glyma.08G351000.1 & 8 & 1644 & 547 & 62 & S & 4 \\
\hline GmPAP27d & Glyma.09G225000.1 & 9 & 1923 & 640 & 71 & S & 5 \\
\hline GmPAP10a & Glyma.09G229200.1 & 9. & 1395 & 464 & 53 & $S$ & 5 \\
\hline GmPAP22b & Glyma.10G071000.1 & 10 & 1266 & 421 & 48 & S & 3 \\
\hline GmPAP15b & Glyma.11G255700.1 & 11 & 1398 & 466 & 52 & $M$ & 7 \\
\hline GmPAP10b & Glyma.12G007500.1 & 12 & 1395 & 464 & 53 & S & 5 \\
\hline GmPAP27e & Glyma.12G012000.1 & 12 & 1980 & 659 & 73 & S & 6 \\
\hline GmPAP10c & Glyma.12G221100.1 & 12 & 1410 & 469 & 54 & S & 4 \\
\hline GmPAP26a & Glyma.13G161900.1 & 13 & 1428 & 475 & 54 & S & 2 \\
\hline GmPAP10d & Glyma.13G280500.1 & 13 & 1416 & 471 & 54 & S & 4 \\
\hline GmPAP18c & Glyma.14G024700.1 & 14 & 1383 & 460 & 52 & S & 1 \\
\hline GmPAP26b & Glyma.17G109400.1 & 17 & 1539 & 512 & 59 & M & 4 \\
\hline GmPAP15c & Glyma.18G001300.1 & 18 & 1719 & 572 & 56 & S & 6 \\
\hline GmPAP1c & Glyma.18G132500.1 & 18 & 1851 & 616 & 69 & S & 3 \\
\hline GmPAP23 & Glyma.19G026600.1 & 19 & 1764 & 587 & 65 & S & 5 \\
\hline GmPAP22c & Glyma.19G193900.1 & 19 & 1473 & 490 & 55 & C & 1 \\
\hline GmPAP20b & Glyma.19G194000.1 & 19 & 1290 & 429 & 48 & $S$ & 1 \\
\hline GmPAP17c & Glyma.20G011900.1 & 20 & 984 & 327 & 37 & S & 3 \\
\hline GmPAP9 & Glyma.20G026800.1 & 20 & 1989 & 662 & 74 & $S$ & 6 \\
\hline
\end{tabular}

For predicted subcellular location, "S", "C", "M", and "-" mean secretory, chloroplast, mitochondrial, and any other location, respectively.

adenosine monophosphate (AMP), glucose-6-phosphate (G-6$\mathrm{P})$, glycerol-2-phosphate (G-2-P), guanosine monophosphate (GMP), inosine monophosphate (IMP), phospho-threonine (PThr), phospho-serine (P-Ser), and $\rho$-NPP. The relative activities were shown as the amount of Pi released from different substrates against the Pi released from $\rho$-NPP as the substrate. To determine the effects of different metal ions on GmPAP7a/7b activities, a total of nine different metal ions were applied at a final concentration of $5 \mathrm{mM}: \mathrm{Mg}^{2+}, \mathrm{Fe}^{2+}, \mathrm{Al}^{3+}, \mathrm{Mn}^{2+}, \mathrm{Zn}^{2+}, \mathrm{Cu}^{2+}$, $\mathrm{Ba}^{2+}, \mathrm{Ca}^{2+}$, and $\mathrm{Co}^{2+}$. The metal ions and GmPAP7a/7b proteins were separately incubated in reaction mixtures which consisted of $45 \mathrm{mM} \mathrm{Na}$-acetate buffer ( $\mathrm{pH} 5.0$ ) and $5 \mathrm{mM}$ $\rho$-NPP to analyze their activities. The relative activities were expressed as the activities with different metal ions divided by the activities without the addition of metal ions. Furthermore, the $V_{\max }$ and $K_{\mathrm{m}}$ values of GmPAP7a/7b toward ATP substrate were determined using a Lineweaver-Burk double reciprocal plot at different ATP concentrations. The units were expressed as the amount of Pi released per minute per milligram protein. All the data are shown as the means of three independent experiments.

\section{Functional Analysis of GmPAP7a/7b in Soybean Hairy Roots}

The open reading frame of $G m P A P 7 a / 7 b$ was separately amplified using specific primers (GmPAP7a/7b-OX-F/R) and subsequently cloned into a $p$ TF101s binary vector. This enables glufosinate ammonium selection for positive transformants. Agrobacterium 


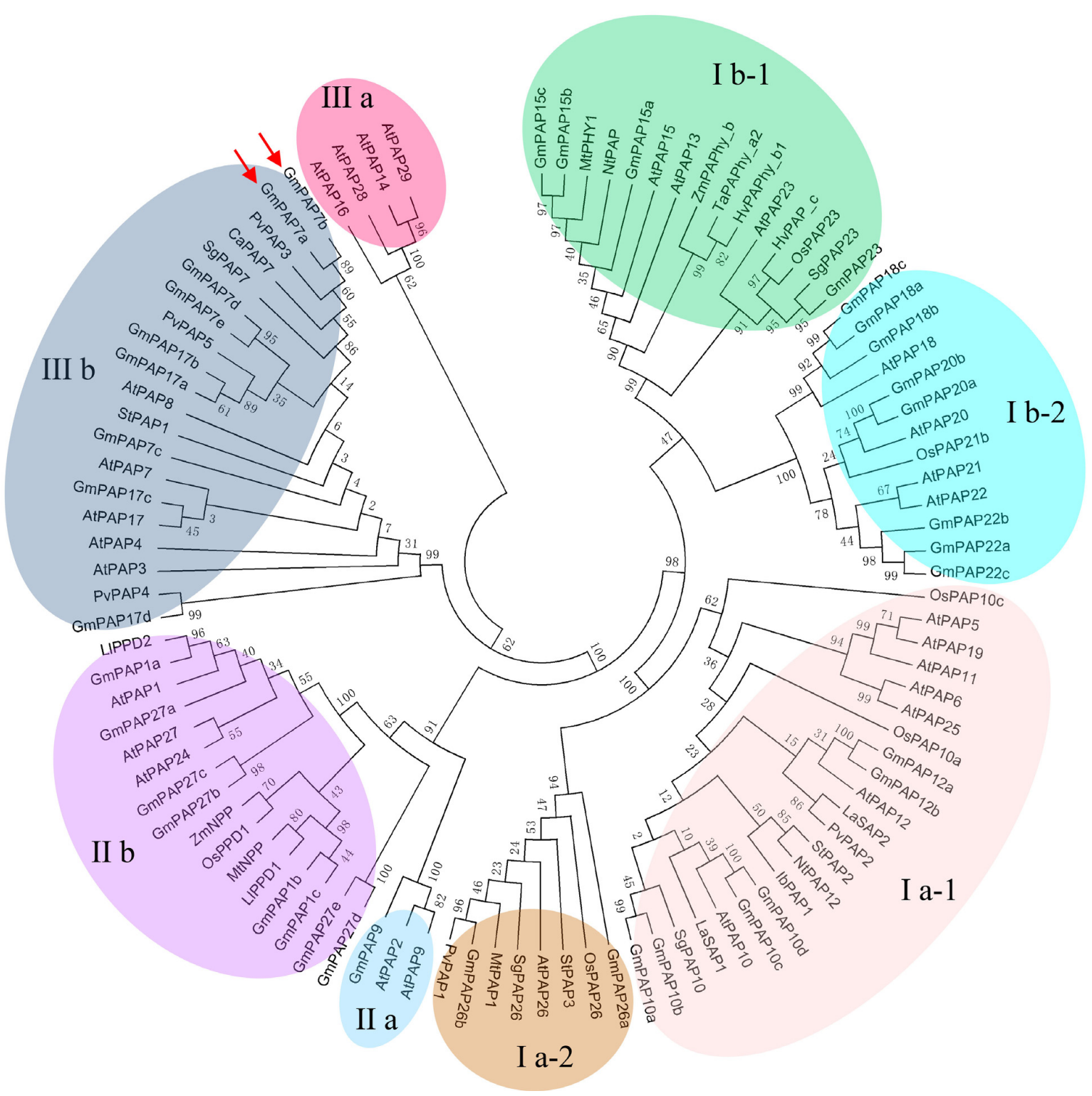

FIGURE 3 | Phylogenetic analysis of PAPs in different plant species. The first two letters of each PAP indicate the abbreviated species name. At: Arabidopsis thaliana, Gm: Glycine max, Pv: Phaseolus vulgaris, Os: Oryza sativa, Zm: Zea mays, Ll: Lupinus luteus, Ta: Triticum aestivum, Nt: Nicotiana tabacum, Hv: Hordeum vulgare, Sg: Stylosanthes guianensis, Mt: Medicago truncatula, La: Lupinus albus, St: Solanum tuberosum, Ib: Ipomoea batatas, Ca: Cicer arietinum. The phylogenetic tree was constructed by MEGA 5.01 program using the neighbor-joining method, with 1000 bootstrap replicates. The bootstrap values are indicated for major branches as percentages. GmPAP7a and GmPAP7b are indicated by red arrows.

rhizogenes-mediated soybean hairy root transformation was performed as described previously (Wu et al., 2018). Briefly, YC03-3 seeds were surface sterilized and germinated on halfstrength MS media for 4 days. The cotyledons were wounded and then transferred to MS media containing $100-200 \mu \mathrm{g} \mathrm{L}^{-1}$ glufosinate ammonium and $500 \mu \mathrm{g} \mathrm{mL}^{-1}$ carbenicillin disodium. After 14-20 days of growth, 18 independent transgenic lines, including nine overexpression lines and nine empty controls (verified by qRT-PCR), were selected for ATP utilization analysis as described previously (Liang et al., 2010). Briefly, approximately $0.1 \mathrm{~g}$ of transgenic soybean hairy roots was transferred to new solid MS media containing $0.4 \mathrm{mM}$ ATP (Sigma-Aldrich,

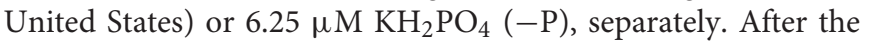
root grew for 14 days, intercellular and root-associated APase activities, root fresh weight, and total $\mathrm{P}$ content were measured.

\section{Statistical Analyses}

Data analyses and standard error calculations were statistically performed using Microsoft Excel 2016 (Microsoft Company, United States), and $t$-tests were performed with the SPSS program (v21.0; SPSS Institute, United States). 


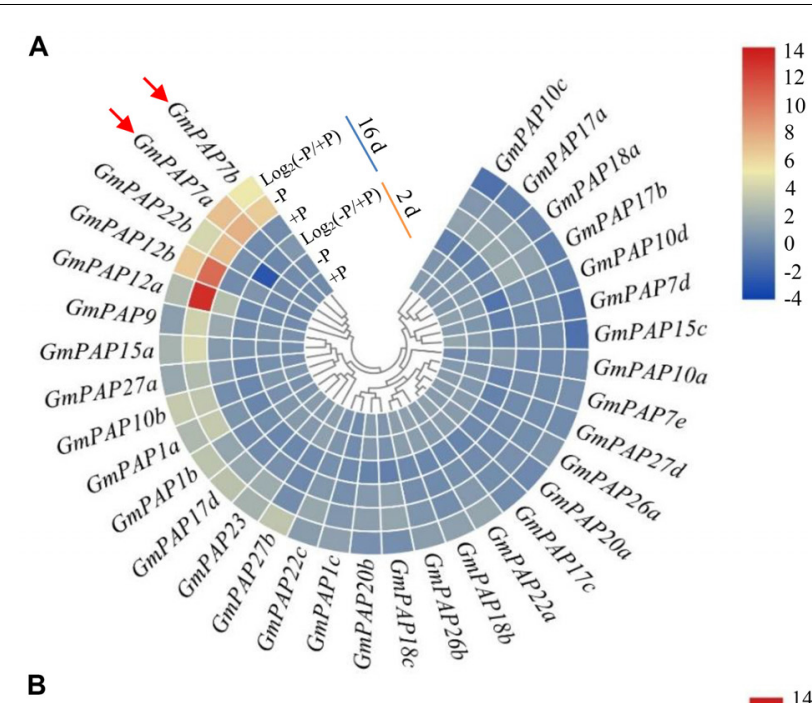

B

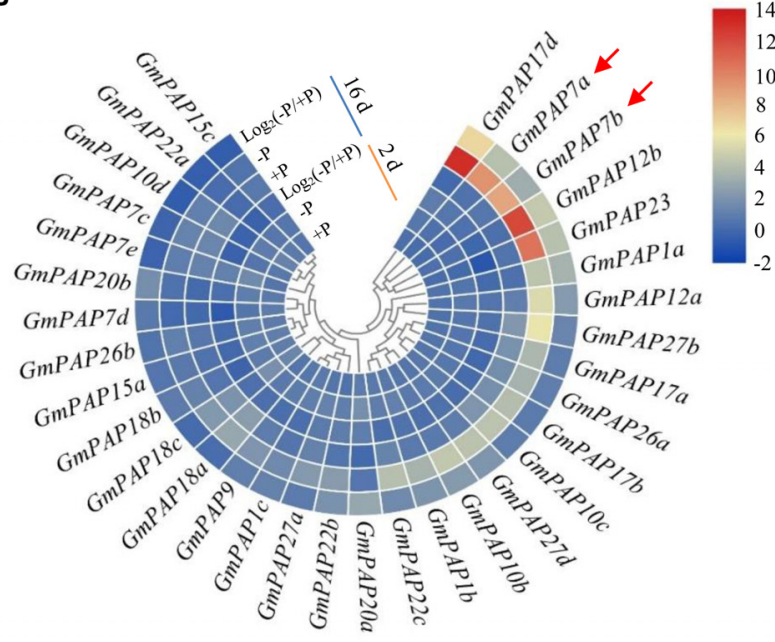

FIGURE 4 | Heatmap analysis of the expression patterns of GmPAP members in leaves and roots in response to $\mathrm{P}$ deficiency. (A) Leaves. (B) Roots. Soybean seedlings were grown in nutrient solution supplemented with $5 \mu \mathrm{M}$ $(-\mathrm{P})$ or $250 \mu \mathrm{M}(+\mathrm{P}) \mathrm{KH}_{2} \mathrm{PO}_{4}$. The leaves and roots were harvested for qRT-PCR analysis after 2 and 16 days of $P$ treatment. $\log _{2}(-P /+P)$ represents the binary logarithm of the fold changes of the relative expression of GmPAP members under Pi-deficient and Pi-sufficient conditions. The data are the means of four replicates. GmPAP7a and GmPAP7b are indicated by red arrows.

\section{RESULTS}

\section{Dynamic Effects of Pi Starvation on Soybean Biomass and P Concentration}

To investigate the effects of low Pi availability on soybean growth, the fresh weight of soybean shoots and roots and the total $\mathrm{P}$ concentration in both the leaves and roots were determined on different days after two P treatments. Although both shoot and root fresh weight increased during the period of the two $\mathrm{P}$ treatments, Pi availability exhibited different effects on dynamic changes in shoot and root fresh weight (Figures 1A,B). For shoot fresh weight, there was no difference between the $+\mathrm{P}$ and
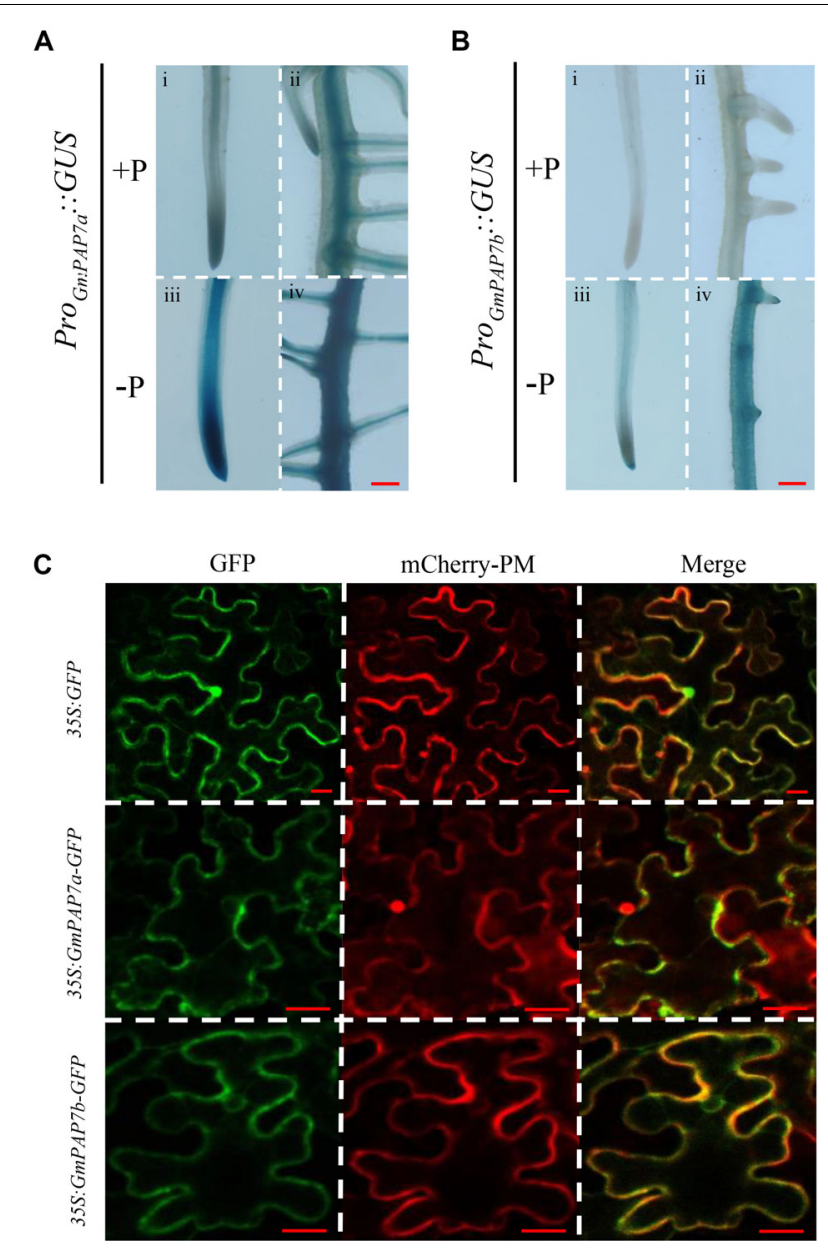

FIGURE 5 | Histochemical and subcellular localization of GmPAP7a/7b. (A) GUS staining of transgenic soybean hairy roots harboring the GmPAP7a promoter (i-iv). (B) GUS staining of transgenic soybean hairy roots harboring the GmPAP7b promoter (i-iv). Transgenic soybean hairy roots were grown on MS media supplemented with $1250(+\mathrm{P}) \mu \mathrm{M}$ or $5 \mu \mathrm{M}(-\mathrm{P}) \mathrm{KH}_{2} \mathrm{PO}_{4}$ for 7 days, and then incubated with GUS staining for further analysis. Bars $=50 \mu \mathrm{m}$.

(C) Subcellular localization of GmPAP7a and GmPAP7b. Transient expression of empty vector control (35S:GFP) or 35S:GmPAP7a/7b-GFP constructs in tobacco leaf epidermal cells. Both GFP and mCherry-PM fluorescence were observed using confocal microscopy. mCherry-PM indicated the plasma membrane AtPIP2A-mCherry marker. Bars $=20 \mu \mathrm{m}$.

$-\mathrm{P}$ treatments during the early period of $\mathrm{P}$ treatments (i.e., $0-$ 10 days), but shoot fresh weight under $-\mathrm{P}$ conditions was $10 \%$ and $17 \%$ less than that under $+\mathrm{P}$ conditions at 13 days and 16 days, respectively (Figure 1A), suggesting that Pi starvation significantly inhibited shoot growth after 13 days of $\mathrm{P}$ treatment. However, root fresh weight increased in response to Pi starvation at 10 days and reached the highest at 16 days, which was 99\% more than that under $+\mathrm{P}$ conditions (Figure 1B), suggesting that Pi starvation enhanced soybean root growth. In contrast, significant decreases in total $\mathrm{P}$ concentration were observed in both the leaves and roots in response to Pi starvation, as reflected by the $48.1 \%$ decrease in the leaves at 5 days and the $46.1 \%$ decrease in the roots at 1 day (Figures 1C,D). Furthermore, the 
ratios of leaf $\mathrm{P}$ concentration at low $\mathrm{P}$ levels to that at high $\mathrm{P}$ levels were approximately 0.9 at 4 days and 0.3 at 16 days, but the ratios in the roots were 0.6 at 4 days and 0.2 at 16 days (Figures 1C,D). This suggests that Pi homeostasis in roots seems more susceptible to $\mathrm{Pi}$ availability than that in the leaves.

\section{Phosphate Starvation Increases APase Activities in the Leaves and Roots}

Dynamic changes in intracellular and root-associated APase activities were further investigated in both leaves and roots at two P levels. Distinct changes in APase activities were observed between leaves and roots under two P conditions. In the leaves, intracellular APase activities reached their highest levels at 4 and 5 days under low- and high-P conditions, respectively, followed by significant decreases with increased duration of $\mathrm{P}$ treatment (Figure 2A). Furthermore, leaf intracellular APase activities at low $\mathrm{P}$ levels were significantly higher than those at high $\mathrm{P}$ levels at 4, 13, and 16 days (Figure 2A), strongly suggesting that low Pi availability significantly affected intracellular APase activities in the leaves. Unlike changes in leaf intracellular APase activities, root intracellular APase activities were significantly enhanced at 1 day and increased by approximately 7 -fold at 16 days in response to by Pi starvation (Figure 2B). Furthermore, root intracellular APase activities were gradually enhanced with increased duration of $\mathrm{Pi}$ starvation, but they remained unchanged after 5 days of high $\mathrm{P}$ treatment (Figure 2B). Although changes of root-associated APase activities exhibited a similar trend between the two P levels, Pi starvation led to more increases of root-associated APase activities after 10 days of $\mathrm{P}$ treatment (Figure 2C). Especially at 16 days, root-associated APase activities at low $\mathrm{P}$ levels were approximately 4-fold higher than those at high P levels (Figure 2C). When BCIP served as the substrate, root-associated APase activities were also visualized. A more intense blue color was detected on root surface under low-P conditions than that under high-P conditions, especially at 10,13 , and 16 days (Figure $2 \mathrm{C}$ ), strongly suggesting that $\mathrm{Pi}$ starvation enhanced root-associated APase activities in soybean.

\section{Bioinformatic and Phylogenetic Analyses}

When sequence homology alignment and conserved PAP domain analysis were used, compared to the 35 GmPAP members identified previously (Li C. C. et al., 2012), five new GmPAP members were identified, and two GmPAP members were not found in the updated soybean genome database. Therefore, a total of $38 \mathrm{GmPAP}$ members were annotated and named in terms of sequence homology with corresponding AtPAP members by using multiple alignments and phylogenetic analysis. The general information of GMPAP members, including chromosomal location, length of coding sequence (CDS), and protein mass, is summarized in Table 1. The GMPAP members were found to be localized on different chromosomes (Table 1). The protein mass of the GmPAP family varied from $35 \mathrm{kD}$ (GmPAP7d) to $74 \mathrm{kD}$ (GmPAP9), and the full length of the CDS and number of amino acids varied from 933 to $1,989 \mathrm{bp}$, and from 310 to 662 aa, respectively (Table 1). Most GmPAP members were predicted to be involved in the secretory pathway. However,
GmPAP22a and GmPAP22c were localized in the chloroplasts; GmPAP26b, GmPAP15b, and GmPAP27c in the mitochondria; and GmPAP1a, GmPAP12a, and GmPAP18b in other subcellular organelles (Table 1). Moreover, all GmPAP members except GmPAP27c and GmPAP7d were predicted to be modified by glycans (Table 1).

An unrooted phylogenetic tree was constructed by MEGA 5.01 using the neighbor-joining method; PAPs were identified in various plant species, including common bean, rice, Arabidopsis, sweet potato, soybean, maize, lupinus, wheat, tobacco, barely, stylo, Medicago, white lupin, potato, and chickpea (Figure 3). It was observed that all plant PAPs could be mainly divided into three main groups comprising eight subgroups, all of which encompassed GmPAP members except subgroup III a (Figure 3). The molecular weights of the plant PAPs in group I and group II were approximately $55 \mathrm{kD}$ and $75 \mathrm{kD}$, respectively, which were higher than those in the group III, of which the PAPs had a molecular weight of approximately $35 \mathrm{kD}$ (Table 1 and Figure 3). Twenty out of $38 \mathrm{GmPAP}$ members were assigned to group I. Notably, nine GmPAP members (GmPAP1a, 1b, 1c, 9, $27 \mathrm{a}, 27 \mathrm{~b}, 27 \mathrm{c}, 27 \mathrm{~d}$, and 27e) were clustered into group II, and nine GmPAP members (GmPAP7a, 7b, 7c, 7d, 7e, 17a, 17b, 17c, and $17 \mathrm{~d}$ ) belonged to subgroup III b (Figure 3). Furthermore, GmPAP7a and GmPAP7b were close to PvPAP3 in common bean (Figure 3), which was reported to be involved in extracellular ATP utilization.

\section{Transcripts of GmPAPs in Response to Pi Starvation}

Relative expression levels of GMPAP members in the leaves and roots were analyzed at 2 and 16 days at two P levels through qRT-PCR analysis. Transcripts of all GmPAP members were detectable at both $\mathrm{P}$ levels, except for those of four members in the leaves (i.e., GmPAP27c, GmPAP27e, GmPAP7c, and $G m P A P 15 b)$ and for those of five in the roots (i.e., GmPAP10a, $G m P A P 15 b, G m P A P 17 c, G m P A P 27 c$, and GmPAP27e) (Figure 4 and Supplementary Table S2). At 2 days of $P$ treatment, transcripts of the detected GmPAP members were not influenced by $\mathrm{Pi}$ availability in either the leaves or the roots (Figure 4 and Supplementary Table S2), suggesting that GmPAP members exhibited no response to early $\mathrm{Pi}$ starvation in soybean. However, at 16 days of $\mathrm{P}$ treatments, the transcript levels of nineteen genes (GmPAP1a, 1b, 1c, 7a, 7b, 9, 10b, 12a, $12 b, 15 a, 17 d, 18 b, 22 a, 22 b, 22 c, 23,26 b, 27 a$, and $27 b$ ) were enhanced by more than 1 -fold in the leaves of plants under Pi-deficient conditions compared with those under Pisufficient conditions (Figure $\mathbf{4 A}$ and Supplementary Table S2). Furthermore, five GmPAP members (GmPAP7a, GmPAP7b, GmPAP12a, GmPAP12b, and GmPAP22b) exhibited the highest expression levels in the leaves at low $\mathrm{P}$ level and clustered together (Figure $\mathbf{4 A}$ and Supplementary Table S2). In the roots, transcripts of $17 \mathrm{GmPAP}$ members (GmPAP1a, $1 b, 1 c$, $7 a, 7 b, 10 b, 10 c, 12 a, 12 b, 17 b, 17 d, 20 a, 22 b, 26 a, 23,27 b$, and $27 d$ ) were found to be significantly upregulated under Pi-deficient conditions (Figure 4B and Supplementary Table S2). Furthermore, five GmPAP members (GmPAP7a, GmPAP7b, 

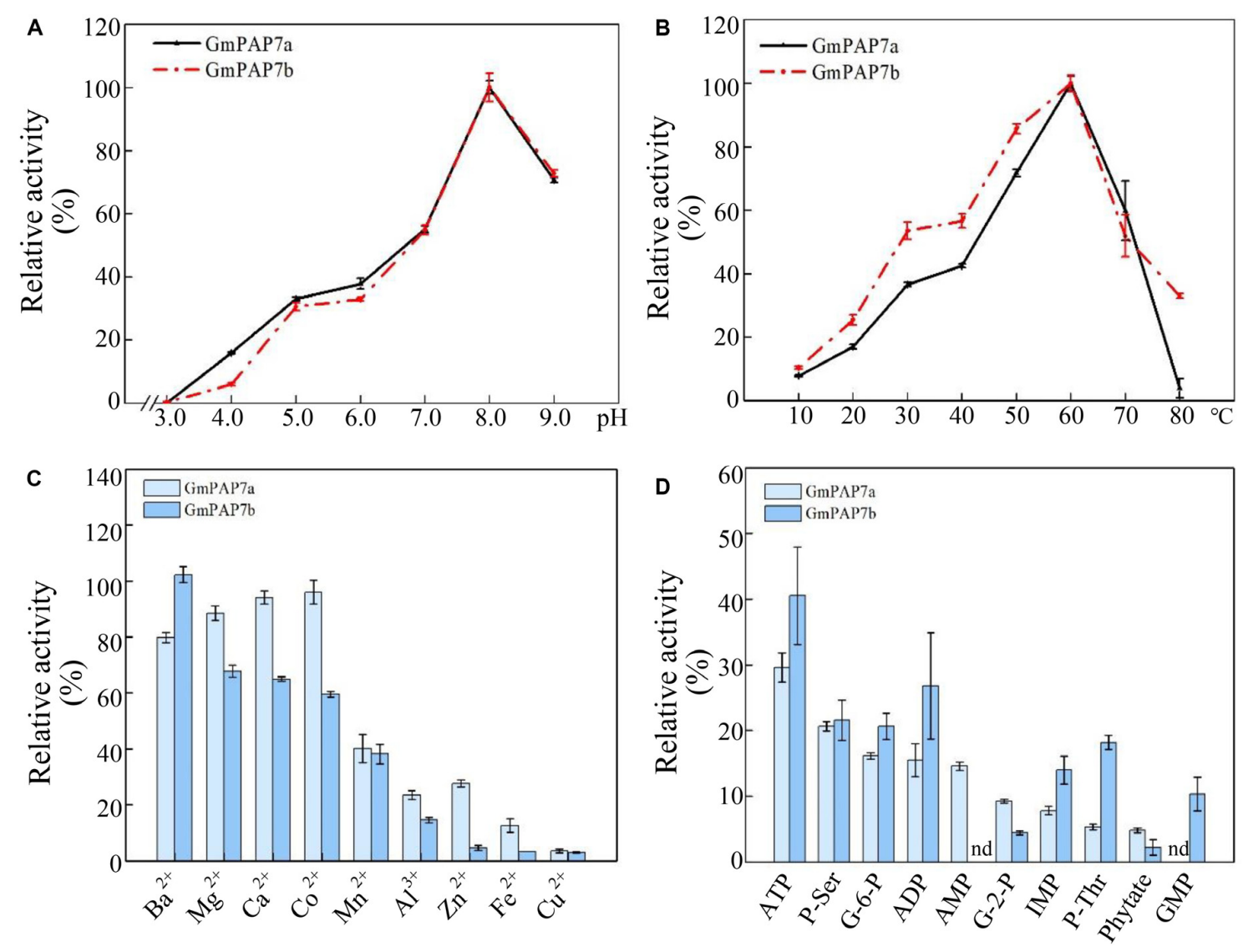

FIGURE 6 | Enzymatic properties of GmPAP7a and GmPAP7b. (A) Effects of pH on the relative activities of GmPAP7a and GmPAP7b. (B) Effects of temperature on the relative activities of GmPAP7a and GmPAP7b. (C) Effects of metal ions on the relative activities of GmPAP7a and GmPAP7b. (D) Relative activities of GmPAP7a and GmPAP7b against different substrates. The data are the means of three replicates with standard errors. nd indicates that no activity was detected.

GmPAP12b, GmPAP17d, and GmPAP23) exhibited the highest expression levels in the roots at low $\mathrm{P}$ level (Figure $\mathbf{4 B}$ and Supplementary Table S2). Overall, the expression levels of 12 GmPAP members (GmPAP1a, 1b, 1c, 7a, 7b, 10b, 12a, 12b, 17d, $22 b, 23$, and $27 b$ ) were observed to be commonly increased in response to $\mathrm{Pi}$ starvation in both the leaves and roots, and this was especially the case for GmPAP7a, GmPAP7b, and GmPAP12b (Figure 4 and Supplementary Table S2).

\section{Tissue Expression Patterns and Subcellular Localization of GmPAP7a/7b}

To further determine the tissue-specific expression patterns of GmPAP7a/7b in response to $\mathrm{Pi}$ starvation, Pro $\mathrm{GmPAP7a}_{\mathrm{a}}$ GUS and $P$ Pro ${ }_{\mathrm{GmPAP}} \mathrm{b}: G U S$ constructs were separately generated and introduced into soybean hairy roots subjected to $\mathrm{P}$ deficiency. Pi starvation led to higher GUS activity in both transgenic soybean hairy roots (Figures 5A,B), suggesting that $\mathrm{Pi}$ starvation could enhance GmPAP7a/7b transcript levels in the roots. Furthermore, under high $\mathrm{P}$ conditions, GUS activity was detected in both the root tips and steles of soybean hairy roots transformed with Pro $_{\text {GmPAP7a:GUS }}$ and only in the stele for Pro ${ }_{\mathrm{GmPAP}} \mathrm{b}$ :GUS (Figures $\mathbf{5 A}, \mathbf{B}$ ), suggesting that different tissue expression patterns might be present between GmPAP7 $a$ and GmPAP7b in roots under high $\mathrm{P}$ conditions. However, under low $\mathrm{P}$ conditions, GUS activity was separately detected in the whole roots transformed with two constructs (Figures $\mathbf{5 A}, \mathbf{B}$ ), suggesting that GmPAP7a and $G m P A P 7 b$ exhibited similar expression patterns in roots at low $\mathrm{P}$ level.

To investigate their subcellular localization, GmPAP7a and GmPAP7b were separately fused to GFP and transiently over-expressed in tobacco leaves. The GFP signals of both 35S:GmPAP7a-GFP and 35S:GmPAP7b-GFP were detected mainly in the plasma membrane and cytoplasm (Figure 5C). However, the GFP signals of the control (35S:GFP) were detected throughout whole cells, including the plasma membrane, cytoplasm, and nucleus (Figure 5C). The results suggested that both GmPAP7a and GmPAP7b were located predominantly in the plasma membrane and cytoplasm (Figure 5C).

\section{Biochemical Characterization of GmPAP7a/7b}

To determine the enzymatic properties of GmPAP7a/7b, recombinant proteins GmPAP7a-GST and GmPAP7b-GST were 


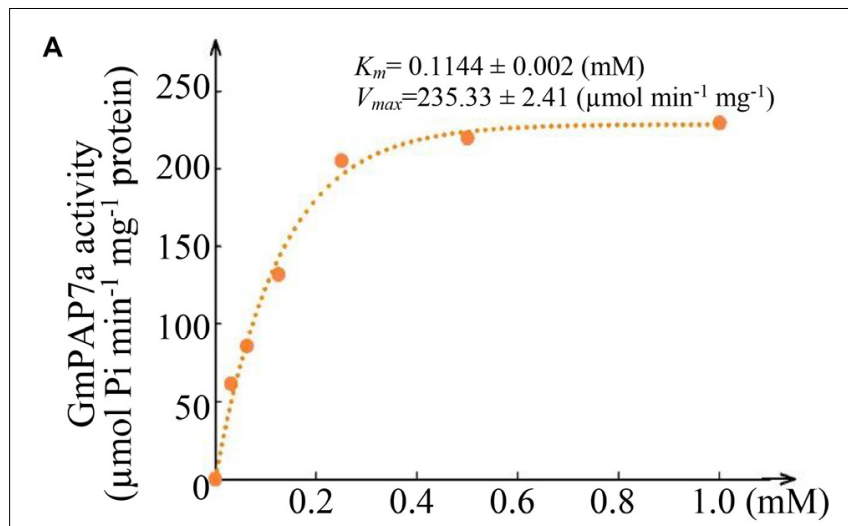

B

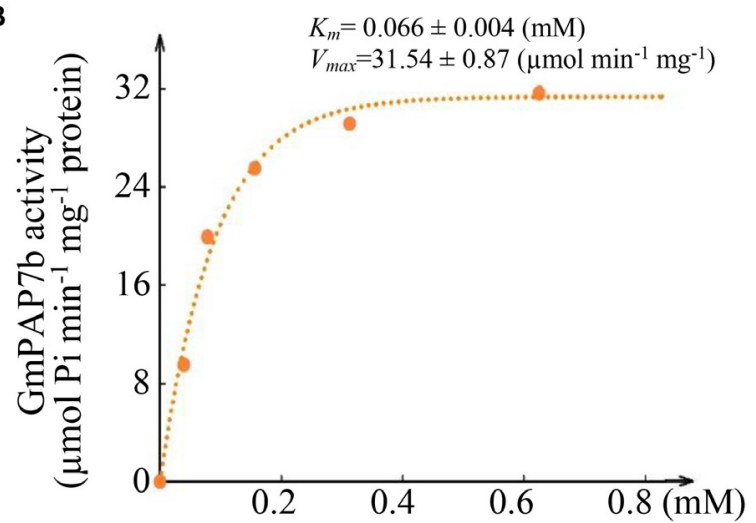

FIGURE 7 | Kinetic parameters of GmPAP7a and GmPAP7b against ATP. (A) Kinetic parameter of GmPAP7a. (B) Kinetic parameter of GmPAP7b. The $K_{m}$ and $V_{\max }$ of GmPAP7a and GmPAP7b were calculated from

Lineweaver-Burke plots of enzyme activities at different concentrations of ATP. The data are the means of three replicates.

separately expressed and successfully purified from E. coli lysates (Supplementary Figure S1). The optimum $\mathrm{pH}$ and temperature for GmPAP7a/7b catalytic reactions were analyzed in vitro using $\rho$-NPP as the substrate, and it was observed that both GmPAP7a and GmPAP7b exhibited similar properties for optimum $\mathrm{pH}$ and temperature, as reflected by the maximum relative activities that were separately observed at $\mathrm{pH} 8.0$ and $60^{\circ} \mathrm{C}$ (Figures 6A,B). However, GmPAP7a activities seem more sensitive to high temperature than GmPAP7b because the relative activities of GmPAP7a and GmPAP7b were 2\% and $30 \%$ at $80^{\circ} \mathrm{C}$, respectively (Figure 6B). In addition, the effects of metal ions on the activities of GmPAP7a and GmPAP7b were separately investigated (Figure 6C). The relative activities of both GmPAP7a and GmPAP7b were reduced by more than $50 \%$ by application of $\mathrm{Mn}^{2+}, \mathrm{Al}^{3+}$, $\mathrm{Zn}^{2+}, \mathrm{Fe}^{2+}$, and $\mathrm{Cu}^{2+}$, especially $\mathrm{Cu}^{2+}$, which inhibited their relative activities by $90 \%$ (Figure $6 \mathrm{C}$ ). However, significant inhibition of relative activities was observed only for GmPAP7b and not for GmPAP7a, when $\mathrm{Mg}^{2+}, \mathrm{Co}^{2+}$ or $\mathrm{Ca}^{2+}$ was applied (Figure 6C).

In a test of substrate specificity, the relative activities of both GmPAP7a and GmPAP7b were highest against
ATP, followed by phosphate-serine for GmPAP7a and ADP for GmPAP7b (Figure 6D). Furthermore, activities against AMP were observed only for GmPAP7a, not for GmPAP7b. Similarly, activities against GMP were observed only for GmPAP7b and not for GmPAP7a (Figure 6D). When ATP served as the substrate, kinetic analyses showed that $K_{\mathrm{m}}$ and $V_{\max }$ values were $0.11 \mathrm{mM}$ and $235.3 \mu \mathrm{mol}$ $\mathrm{min}^{-1} \mathrm{mg}^{-1}$ protein for GmPAP7a and $0.066 \mathrm{mM}$ and $31.5 \mu \mathrm{mol} \mathrm{min} \mathrm{mg}^{-1}$ protein for GmPAP7b, respectively (Figure 7), suggesting that the catalytic properties differ between GmPAP7a and GmPAP7b.

\section{Overexpressing GmPAP7a/7b Enhanced Exogenous ATP Utilization}

To investigate the functions of GmPAP7a and GmPAP7b in exogenous ATP utilization, transgenic soybean hairy roots overexpressing GmPAP7a or GmPAP7b were generated (Figure 8). The increased expression levels of GmPAP7a or $G m P A P 7 b$ in transgenic hairy root lines (OX-GmPAP7a/7b) were verified through qRT-PCR analysis, as reflected by more than 11-fold increases compared to those of the control (CK) lines (Supplementary Figure S2a). Moreover, overexpression of GmPAP7a and GmPAP7b led to significant increases in intracellular APase activities (Supplementary Figure S2b) and root-associated APase activities under P-deficient conditions and ATP application (Figure 8). Furthermore, the fresh weight and the total $\mathrm{P}$ content were approximately $50 \%$ and $28 \%$ higher, respectively, in the soybean hairy root lines with GmPAP7a or GmPAP7b overexpression than in the control lines with ATP application, while no difference was observed in the $-\mathrm{P}$ treatments (Figure 8), strongly suggesting that GmPAP7a and GmPAP7b might participate in extracellular ATP utilization in soybean.

\section{DISCUSSION}

Phosphorus is an essential macronutrient participating in many biochemical and metabolic processes (Liang et al., 2010; Zhang et al., 2014; Ham et al., 2018). Low Pi availability imposes serious limitations on crop growth and production (Vance et al., 2003; Zhang et al., 2014; Dissanayaka et al., 2018). It is generally observed that $\mathrm{P}$ deficiency alters root system to increase the root surface area and exploitable soil volume for Pi uptake (Pérez-Torres et al., 2008; Lambers et al., 2011; Lynch, 2011). In this study, enhanced soybean root growth was also observed in soybean in response to Pi starvation, as reflected by increased soybean root fresh weight after 10 days of Pi starvation (Figure 1), strongly suggesting that significant changes in the soybean root system occur under P-deficient conditions.

In addition to changes in root morphology and architecture, Pi starvation also results in increases in intracellular and extracellular APase activities in plants (Zimmermann et al., 2004; Plaxton and Lambers, 2015; Tian and Liao, 2015; Wang and Liu, 2018). Furthermore, accumulating evidence has suggested that 

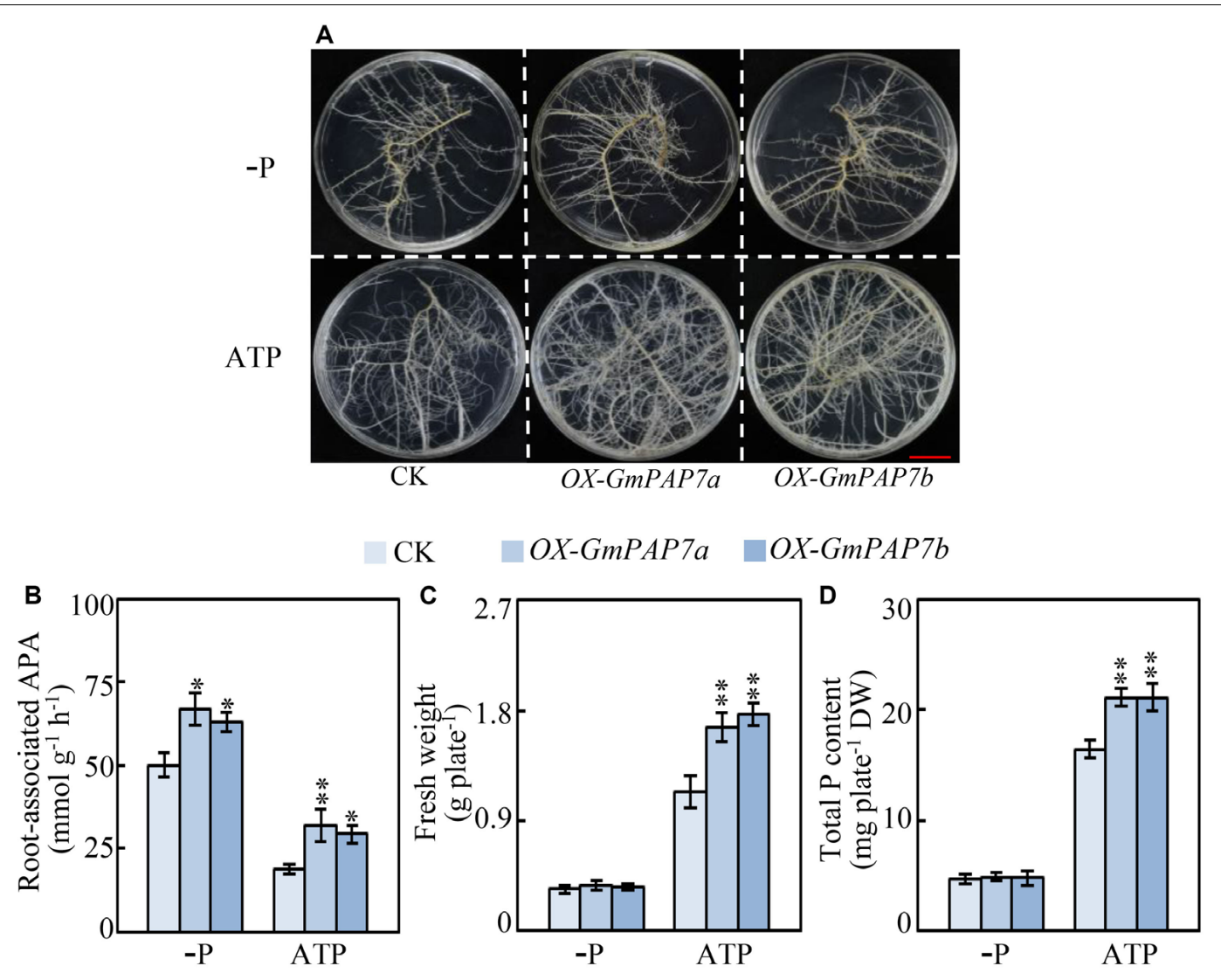

FIGURE 8 | Effects of GmPAP7a and GmPAP7b overexpression on APase activities, total P content and fresh weight of transgenic soybean hairy roots. (A) Image of transgenic hairy roots supplied with different P sources. Bars $=1 \mathrm{~cm}$. (B) Root-associated APase activities. (C) Fresh weight of transgenic soybean hairy roots.

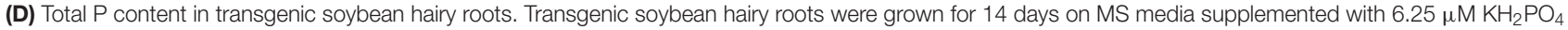
$(-P)$ or 0.4 mM ATP as the sole $P$ source. Fresh weight and total $P$ content were separately measured. CK represents transgenic hairy root lines transformed with an empty vector. OX-GmPAP7a and OX-GmPAP7b indicate transgenic hairy root lines overexpressing GmPAP7a and GmPAP7b, respectively. The data are the means of nine replicates with standard errors. The asterisks indicate significant differences between the overexpression and CK lines according to Student's $t$-test: $* P<0.05,{ }^{* *} P<0.01$.

increases in APase activities are mainly due to an enhanced $P A P$ transcripts under low $\mathrm{P}$ conditions in plants, such as $P v P A P 3$ in bean, AtPAP10/12 in Arabidopsis, OsPAP10a and OsPAP21b in rice (Liang et al., 2010; Tran et al., 2010b; Wang et al., 2011; Tian et al., 2012a; Mehra et al., 2017). In this study, root intracellular and associated APase activities were significantly enhanced by $\mathrm{Pi}$ starvation at 16 days (Figure 2), accompanied by significantly increased transcripts of $17 \mathrm{GmPAP}$ members in the roots (Figure 4B and Supplementary Table S2), strongly suggesting that increased transcripts of GmPAP members could lead to enhanced APase activities in soybean. However, increases of root intracellular APase activities were also detected after 2 days of Pi starvation, while the transcript abundance of no GmPAP member changed in response to Pi starvation in either the leaves or roots (Figures 2, 4), suggesting that there must be other molecular mechanisms underlying APase activity regulation in addition to controlling the transcript levels of GMPAP members. In soybean, 36 out of $38 \mathrm{GmPAP}$ members were predicted to exhibit more than one glycosylation site (Table 1). Moreover, it has been suggested that the enzymatic properties of plant PAPs could be impacted by their glycosylation (Navazio et al., 2002; Olczak and Olczak, 2007; Tran et al., 2010b). Therefore, it is plausible that glycosylation modification of GmPAP members may play a role in regulating APase activities in response to 2 days of $\mathrm{Pi}$ starvation, which merits further analysis.

With the aid of genome sequence availability in various plant species, increased transcripts of PAP members in response to $\mathrm{Pi}$ starvation have been found in plant species, such as Arabidopsis, rice, maize, and chickpea (Zhang et al., 2010; Wang et al., 2014; Gonzalez-Munoz et al., 2015; Bhadouria et al., 2017). Similarly, the expression levels of 19 and 17 GmPAP members increased in response to $\mathrm{Pi}$ starvation in the leaves and roots, respectively (Figure 4 and Supplementary Table S2), suggesting that increased PAP transcription is a common response to $\mathrm{Pi}$ starvation in plants. Moreover, a group of plant PAP members have been suggested to be downstream genes of PHR1 (or its homologs), which is the central regulator in the $\mathrm{P}$ signaling network; these PAPs include AtPAP17 in Arabidopsis, 10 OsPAP members in rice, and PvPAP3 in bean (Rubio et al., 2001; Bari et al., 2006; Zhang et al., 2010; Yao Z. F. et al., 2014). 
These findings suggest that GmPAP members might also be regulated by $P H R 1$ or its homologs in soybean. Consistently, GmPAP14 (i.e., GmPAP7e) and GmPAP21 (i.e., GmPAP22b) have been suggested to be downstream genes of GmPHR25 because GmPHR25 overexpression leads to increased transcript levels in roots of GmPHR25 overexpression composite plants (Xue et al., 2017). However, the regulatory mechanisms of other Pi starvation-responsive GmPAP members remain unclear.

Root-associated PAPs are well known to play a role in the utilization of extracellular organic $\mathrm{P}$ sources, such as ATP, phytate-P and dNTPs (Liang et al., 2010, 2012; Wang et al., 2011, 2014; Robinson et al., 2012; Liu et al., 2016, 2018; Lu et al., 2016; Gao et al., 2017; Mehra et al., 2017; Wu et al., 2018). For example, AtPAP10/12/26 are suggested to participate in the utilization of extracellular DNA and ADP in Arabidopsis (Wang et al., 2011, 2014). However, the functions of PvPAP3 in bean and OsPAP $10 \mathrm{a} / 21 \mathrm{~b} / 26 / 10 \mathrm{c}$ in rice are suggested to mediate extracellular ATP utilization (Liang et al., 2010; Tian et al., 2012a; Lu et al., 2016; Gao et al., 2017; Mehra et al., 2017). In this study, phylogenetic tree analysis revealed that PvPAP3, GmPAP7a, and GmPAP7b belonged to group III b (Figure 3), suggesting they might have similar functions in plants. Moreover, both GmPAP7a and GmPAP7b exhibited relatively high activities against ATP in vitro (Figure 6D) and localized to the plasma membrane and cytoplasm (Figure 5C), which was similar to that occurred for PvPAP3 (Liang et al., 2010). Furthermore, the fresh weight and total $\mathrm{P}$ content in soybean hairy roots with $G m P A P 7 a$ and $G m P A P 7 b$ overexpression were significantly higher than those of control lines with ATP application (Figure 8), strongly suggesting that GmPAP7a and GmPAP7b could utilize extracellular ATP. Interestingly, $\mathrm{pH}$ optima of GmPAP7a and GmPAP7b activity was 8.0 in vitro (Figure 6A). Similarly, $\mathrm{pH}$ optima of several plant PAP activity was found to be above 7.0 despite how the $\mathrm{pH}$ optima of activity for most plant PAPs is generally below 7.0 (Tran et al., 2010a; Plaxton and Lambers, 2015; Tian and Liao, 2015; Wang and Liu, 2018). For example, $\mathrm{pH}$ optima of PvPAP3 against $\rho$-NPP and KbPAP against ATP was 7.0 or above (Cashikar et al., 1997; Liang et al., 2010). Therefore, these results suggest that some plant PAPs might have functions as an alkaline phosphatase, which merits further study.

Remobilization of internal phosphorylated metabolites (e.g., phosphorylated carbon) is generally considered to be an adaptive strategy plants in responses to $\mathrm{Pi}$ starvation (Chiou and Lin, 2011; Tian et al., 2012b; Plaxton and Lambers, 2015; Abel, 2017). Consistently, decreased accumulation of phosphorylated compounds was widely observed in plants under Pi-deficient conditions (Chiou and Lin, 2011; Tian et al., 2012b; Plaxton and Lambers, 2015; Mo et al., 2019). Given that plant PAPs exhibit activity against a set of phosphorylated metabolites, it is suggested that internal plant PAP might play a role in metabolic processes of internal phosphorylated metabolites (Tian et al., 2012b; Plaxton and Lambers, 2015). In this study, since transcript levels of both GmPAP7a and GmPAP7b were also significantly upregulated in leaves after $16 \mathrm{~d}$ of $\mathrm{P}$ deficiency, it is plausible that GmPAP7a and GmPAP7b might participate in the recycling of internal phosphorylated metabolites in leaves.
In summary, a total of 38 GmPAP members were identified in the soybean genome. However, the transcript levels of 19 and 17 GmPAP members were upregulated by 16 days of $\mathrm{P}$ deficiency in the leaves and roots, respectively. Among them, both GmPAP7a and GmPAP7b had the highest activities against ATP in vitro. Furthermore, overexpressing GmPAP7 $a$ and GmPAP7b resulted in significant increases in root-associated APase activities and total P content in soybean hairy roots when ATP was supplied as the sole $\mathrm{P}$ source. Taken together, these results suggest that Pi starvation responsive GmPAP7a and GmPAP7b may mediate root-associated APase activities and thus control extracellular ATP utilization in soybean in response to $\mathrm{P}$ deficiency.

\section{DATA AVAILABILITY STATEMENT}

All datasets generated for this study are included in the article/Supplementary Material.

\section{AUTHOR CONTRIBUTIONS}

JT and CL conceived and designed the experiments. SZ, MC, and SL performed the experiments. JT, CL, SZ, MC, and YX analyzed the data. JT, CL, SZ, and YX wrote the manuscript. All authors have read and approved the final manuscript.

\section{FUNDING}

This work was supported by the Major Program of Guangdong Basic and Applied Research (2019B030302006), the National Natural Science Foundation of China (31872164 and 31672220), the Integrated Demonstration of Key techniques for the Industrial Development of Featured Crops in Rocky Desertification Areas of Yunnan-Guangxi-Guizhou Provinces (SMH2019-2021), the Guangdong Natural Science Funds for Distinguished Young Scholars (2015A030306034), the Key R\&D Program of Guangdong Province (2019B020219003), and the Research Team Project of the Natural Science Foundation of Guangdong Province (2016A030312009).

\section{SUPPLEMENTARY MATERIAL}

The Supplementary Material for this article can be found online at: https://www.frontiersin.org/articles/10.3389/fpls.2020.00661/ full\#supplementary-material

FIGURE S1 | Analysis of the expression and purification of recombinant GmPAP7a-GST and GmPAP7b-GST proteins.

FIGURE S2 | Analysis of GmPAP7a/7b transcription and intracellular APase activities in transgenic overexpression soybean hairy roots.

TABLE S1 | Primers used for qRT-PCR or vector construction.

TABLE S2 | Relative expression levels of GmPAP members. 


\section{REFERENCES}

Abel, S. (2017). Phosphate scouting by root tips. Curr. Opin. Biotechnol. 39, 168-177. doi: 10.1016/j.pbi.2017.04.016

Bari, R., Datt, P. B., Stitt, M., and Scheible, W. R. (2006). PHO2, microRNA399, and PHR1 define a phosphate-signaling pathway in plants. Plant Physiol. 141, 988-999.

Bhadouria, J., Singh, A. P., Mehra, P., Verma, L., Srivastawa, R., Swarup, K., et al. (2017). Identification of purple acid phosphatases in chickpea and potential roles of CaPAP7 in seed phytate accumulation. Sci. Rep. 7:11012. doi: 10.1038/ s41598-017-11490-9

Bradford, M. M. (1976). A rapid and sensitive method for the quantitation of microgram quantities of protein utilizing the principle of protein-dye binding. Anal. Biochem. 72, 248-254. doi: 10.1016/0003-2697(76)90527-3

Cashikar, A. G., Kumaresan, R., and Rao, N. M. (1997). Biochemical characterization and subcellular localization of the red kidney bean purple acid phosphatase. Plant Physiol. 114, 907-915. doi: 10.1104/pp.114.3.907

Chen, L. Y., Qin, L., Zhou, L., Li, X. X., Chen, Z. J., Sun, L., et al. (2018). A nodulelocalized phosphate transporter GmPT7 plays an important role in enhancing symbiotic N2 fixation and yield in soybean. New Phytol. 221, 2013-2025. doi: 10.1111/nph.15541

Chiou, T. J., and Lin, S. I. (2011). Signaling network in sensing phosphate availability in plants. Annu. Rev. Plant Biol. 62, 185-206. doi: 10.1146/annurevarplant-042110-103849

Conley, D. J., Paerl, H. W., Howarth, R. W., Boesch, D. F., Seitzinger, S. P., Havens, K. E., et al. (2009). Controlling eutrophication: nitrogen and phosphorus. Science 323, 1014-1015. doi: 10.1126/science.1167755

Conner, T., Paschal, E. H., Barbero, A., and Johnson, E. (2004). The challenges and potential for future agronomic traits in soybeans. Agbio. Forum. 7, 47-50.

Del Pozo, J. C., Allona, I., Rubio, V., Leyva, A., De la Pena, A., Aragoncillo, C., et al. (1999). A type 5 acid phosphatase gene from Arabidopsis thaliana is induced by phosphate starvation and by some other types of phosphate mobilizing/oxidative stress conditions. Plant J. 19, 579-589. doi: 10.1046/j. 1365-313x.1999.00562.x

Dionisio, G., Madsen, C. K., Holm, P. B., Welinder, K. G., Jorgensen, M., Stoger, E., et al. (2011). Cloning and characterization of purple acid phosphatase phytases from wheat, barley, maize, and rice. Plant Physiol. 156, 1087-1100. doi: $10.1104 /$ pp.110.164756

Dissanayaka, D. M. S. B., Nishida, S., Tawaraya, K., and Wasaki, J. (2018). Organspecific allocation pattern of acquired phosphorus and dry matter in two rice genotypes with contrasting tolerance to phosphorus deficiency. J. Plant Nutr. Soil Sci. 64, 1-9. doi: 10.1080/00380768.2018.1436941

Gao, W. W., Lu, L. H., Qiu, W. M., Wang, C., and Shou, H. X. (2017). OsPAP26 encodes a major purple acid phosphatase and regulates phosphate remobilization in rice. Plant Cell Physiol. 58, 885-892. doi: 10.1093/pcp/pcx041

Gonzalez-Munoz, E., Avendano-vazquez, A. Q., Chavez-Montes, R. A., de Folter, S., Andres-Hernandez, L., Abreu-Goodger, L. C., et al. (2015). The maize (Zea mays ssp. Mays var B73) genome encodes 33 members of the purple acid phosphatases family. Front. Plant Sci. 6:341. doi: 10.3389/fpls.2015.00341

Ham, B. K., Chen, J. Y., Yan, Y., and William, J. L. (2018). Insights into plant phosphate sensing and signaling. Curr. Opin. Biotechnol. 49, 1-9.

Haran, S., Logendra, S., Seskar, M., Bratanova, M., and Raskin, I. (2000). Characterization of Arabidopsis acid phosphatase promoter and regulation of acid phosphatase expression. Plant Physiol. 124, 615-626. doi: 10.2307/4279464

Herridge, D. F., Peoples, M. B., and Boddey, R. M. (2008). Global inputs of biological nitrogen fixation in agricultural systems. Plant Soil 311, 1-18. doi: 10.1104/pp.126.4.1598

Jefferson, R. A., Kavanagh, T. A., and Bevan, M. W. (1987). GUS fusions: $\beta$ glucuronidase as a sensitive and versatile gene fusion marker in higher plants. EMBO J. 6, 3901-3907.

Jung, J. Y., Riled, M. K., Hothorn, M., and Poirier, Y. (2018). Control of plant phosphate homeostasis by inositol pyrophosphatase and the SPX domain. Curr. Opin. Biotechnol. 49, 156-162.

Kaida, R., Sage-Ono, K., Kamada, H., Okuyama, H., Syono, K., and Kaneko, T. S. (2003). Isolation and characterization of four cell wall purple acid phosphatase genes from tobacco cells. Biochim. Biophys. Acta 1625, 134-140. doi: 10.1016/ S0167-4781(02)00599-7
Kong, Y. B., Li, X. H., Ma, J., Li, W. L., Yan, G. J., and Zhang, C. Y. (2014). GmPAP4, a novel purple acid phosphatase gene isolated from soybean (Glycine max), enhanced extracellular phytate utilization in Arabidopsis thaliana. Plant Cell Rep. 33, 655-667. doi: 10.1007/s00299-014-1588-5

Kong, Y. B., Li, X. H., Wang, B., Li, W. L., Du, H., and Zhang, C. Y. (2018). The soybean purple acid phosphatase GmPAP14 predominantly enhances external phytate utilization in plants. Front. Plant Sci. 9:292. doi: 10.3389/fpls.2018. 00292

Lambers, H., Finnegan, P. M., Laliberté, E., Pearse, S. J., Ryan, M. H., Shane, M. W., et al. (2011). Phosphorus nutrition of proteaceae in severely phosphorusimpoverished soils: are there lessons to be learned for future crops? Plant Physiol. 156, 1058-1066. doi: 10.1104/pp.111.174318

Li, C. C., Gui, S. H., Yang, T., Walk, T., Wang, X. R., and Liao, H. (2012). Identification of soybean purple acid phosphatase genes and their expression responses to phosphorus availability and symbiosis. Ann. Bot. 109, 275-285. doi: $10.1093 / \mathrm{aob} / \mathrm{mcr} 246$

Li, C. C., Zhou, J., Wang, X. R., and Liao, H. (2019). A purple acid phosphatase, GmPAP33, participates in arbuscule degeneration during arbuscular mycorrhizal symbiosis in soybean. Plant Cell Envir. 42, 2015-2027. doi: 10.1111 /pce. 13530

Li, D. P., and Wang, D. W. (2003). cDNA cloning and in vitro expressions of three putative purple acid phosphatase genes from Arabidopsis. J. Nat. Sci. 26, 3-29. doi: 10.1023/A:1022289509702

Li, D. P., Zhu, H. F., Liu, K. F., Liu, X., Leggewie, G., and Udvardi, M. (2002). Purple acid phosphatases of Arabidopsis thaliana. J. Biol. Chem. 31, 27772-27781. doi: 10.1074/jbc.M204183200

Li, R. J., Lu, W. J., Guo, C. J., Li, X. J., Gu, J. T., and Xiao, K. (2012). Molecular characterization and functional analysis of OsPHY1, a purple acid phosphatase (PAP)-type phytase gene in rice (Oryza sativa L.). J. Integr. Plant Biol. 11, 1217-1226.

Li, X. H., Wang, Y. J., Wu, B., Kong, Y. B., Li, W. L., Chang, W. S., et al. (2014). GmPHR1, a novel homolog of the AtPHR1 transcription factor, plays a role in plant tolerance to phosphate starvation. J. Integr. Agric. 13, 2584-2593. doi: 10.1016/S2095-3119(14)60775-9

Li, X. X., Zhao, J., Tan, Z. Y., Zeng, R. S., and Liao, H. (2015). GmEXPB2, a cell wall $\beta$-expansin, affects soybean nodulation through modifying root architecture and promoting nodule formation and development. Plant Physiol. 169, 2640-2653. doi: 10.1104/pp.15.01029

Liang, C. Y., Sun, L. L., Yao, Z. F., Liao, H., and Tian, J. (2012). Comparative analysis of PvPAP gene family and their functions in response to phosphorus deficiency in common bean. PLoS One 7:e38106. doi: 10.1371/journal.pone.003 8106

Liang, C. Y., Tian, J., Lam, H. M., Lim, B. L., Yan, X., and Liao, H. (2010). Biochemical and molecular characterization of PvPAP3, a novel purple acid phosphatase isolated from common bean enhancing extracellular ATP utilization. Plant Physiol. 152, 854-865. doi: 10.1104/pp.109.147918

Liang, C. Y., Wang, J. X., Zhao, J., Tian, J., and Liao, H. (2014). Control of phosphate homeostasis through gene regulation in crops. Curr. Opin. Plant Biol. 21, 59-66. doi: 10.1016/j.pbi.2014.06.009

Liao, H., Wan, H. Y., Shaff, J., Wang, X. R., Yan, X. L., and Kochian, L. V. (2006). Phosphorus and aluminum interactions in soybean in relation to aluminum tolerance. Exudation of specific organic acids from different regions of the intact root system. Plant Physiol. 141, 674-684. doi: 10.1104/pp.105.076497

Liu, L., Liao, H., Wang, X. R., and Yan, X. L. (2008). Regulation effect of soil $\mathrm{P}$ availability on mycorrhizal infection in relation to root architecture and $\mathrm{P}$ efficiency of Glycine max. Chinese J. Appl. Ecol. 19, 564-568.

Liu, P. D., Cai, Z. F., Chen, Z. J., Mo, X. H., and Ding, X. P. (2018). A rootassociated purple acid phosphatase, SgPAP23, mediates extracellular phytate-P utilization in Stylosanthes guianensis. Plant Cell Environ. 41, 2821-2834. doi: $10.1111 /$ pce. 13412

Liu, P. D., Xue, Y. B., Chen, Z. J., Liu, G. D., and Tian, J. (2016). Characterization of purple acid phosphatases involved in extracellular dNTP utilization in Stylosanthes. J. Exp. Bot. 67, 4141-4154. doi: 10.1093/jxb/erw190

Lu, L. H., Qiu, W. M., Gao, W. W., Tyerman, S. D., Shou, H. X., and Wang, C. (2016). OsPAP10c, a novel secreted acid phosphatase in rice, plays an important role in the utilization of external organic phosphorus. Plant Cell Environ. 39, 2247-2259. doi: 10.1111/pce.12794 
Lynch, J. P. (2011). Root phenes for enhanced soil exploration and phosphorus acquisition: tools for future crops. Plant Physiol. 156, 1041-1049. doi: 10.2307/ 41435018

Ma, X. F., Wright, E., Ge, Y. X., Bell, J., Xi, Y. J., and Bouton, J. H. (2009). Improving phosphorus acquisition of white clover (Trifolium repens L.) by transgenic expression of plant-derived phytase and acid phosphatase genes. Plant Sci. 176, 479-488. doi: 10.1016/j.plantsci.2009.01.001

Madsen, C. K., Dionisio, G., Holme, I. B., Holm, P. B., and Henrik, B. P. (2013). High mature grain phytase activity in the Triticeae has evolved by duplication followed by neofunctionalization of the purple acid phosphatase phytase (PAPhy) gene. J. Exp. Bot. 64, 3111-3123. doi: 10.1093/jxb/ert116

Mehra, P., Pandey, B. K., and Giri, J. (2017). Improvement in phosphate acquisition and utilization by a secretory purple acid phosphatase (OsPAP21b) in rice. Plant Biotech. J. 15, 1054-1067. doi: 10.1111/pbi.12699

Mo, X. H., Zhang, M. K., Liang, C. Y., Cai, L. Y., and Tian, J. (2019). Integration of metabolome and transcriptome analyses highlights soybean roots responding to phosphorus deficiency by modulating phosphorylated metabolite processes. Plant Physiol. Biochem. 139, 697-706. doi: 10.1016/j.plaphy.2019.04.033

Murphy, J., and Riley, J. (1963). A modified single solution method for the determination of phosphate in natural waters. Anal. Chim. Acta 27, 31-35. doi: 10.1016/S0003-2670(00)88444-5

Navazio, L., Miuzzo, M., Royle, L., Baldan, B., Varotto, S., Merry, A. H., et al. (2002). Monitoring endoplasmic reticulum-to-Golgi traffic of a plant calreticulin by protein glycosylation analysis. Biochemistry 41, 14141-14149. doi: 10.1021/ bi0204701

Olczak, M., Morawiecka, B., and Watorek, W. (2003). Plant purple acid phosphatases-genes, structures and biological function. Acta. Biochim. Pol. 50, 1245-1256. doi: 10.1016/S1381-1177(02)00143-1

Olczak, M., and Olczak, T. (2007). N-glycosylationsites of plant purple acid phosphatases important for protein expression and secretion in insect cells. Arch. Biochem. Biophys. 461, 247-254. doi: 10.1016/j.abb.2007.02.005

Pérez-Torres, C. A., López-Bucio, J., Cruz-Ramírez, A., Ibarra-Laclette, E., Dharmasiri, S., Estelle, M., et al. (2008). Phosphate availability alters lateral root development in Arabidopsis by modulating auxin sensitivity via a mechanism involving the TIR1 auxin receptor. Plant Cell 20, 3258-3272. doi: 10.1105/tpc. 108.058719

Plaxton, W. C., and Lambers, H. (2015). "Phosphorus: back to the roots," in Annual. Plant Reviews, Volume 48: Phosphorus Metabolism in Plants, eds W. C. Plaxton, and H. Lambers, (Hoboken, NJ: WileyBlackwell), 3-22.

Robinson, W. D., Park, J., Tran, H. T., Del Vecchio, H. A., Ying, S., Zins, J. L., et al. (2012). The secreted purple acid phosphatases isozymes AtPAP12 and AtPAP26 play a pivotal role in extracellular phosphate-scavenging by Arabidopsis thaliana. J. Exp. Bot. 63, 6531-6542. doi: 10.1093/jxb/ers 309

Rubio, V., Linhares, F., Solano, R., Martin, A. C., Lglesias, J., and Leyva, A. (2001). A conserved MYB transcription factor involved in phosphate starvation signaling both in vascular plants and in unicellular algae. Gene Dev. 15, 2122-2133. doi: $10.1101 /$ gad.204401

Tian, J., and Liao, H. (2015). "The role of intracellular and secreted purple acid phosphatases in plant phosphorus scavenging and recycling," in Annual. Plant Reviews, Volume 48, Phosphorus Metabolism in Plants, eds W. C. Plaxton, and H. Lambers, (Hoboken, NJ: WileyBlackwell), 265-287.

Tian, J., Liao, H., Wang, X. R., and Yan, X. L. (2003). Phosphorus starvationinduced expression of leaf acid phosphatase isoforms in soybean. Acta Bot. Sin. $45,1037-1042$.

Tian, J., Wang, C., Zhang, Q., He, X. W., Whelan, J., and Shou, H. X. (2012a). Overexpression of OsPAP10a, a root-associated acid phosphatase, increased extracellular organic phosphorus utilization in rice. J. Integr. Plant Biol. 54, 631-639. doi: 10.1111/j.1744-7909.2012.01143.x

Tian, J., Wang, X. R., Tong, Y., Chen, X., and Liao, H. (2012b). Bioengineering and management for efficient phosphorus utilization in crops and pastures. Curr. Opin. Biotechnol. 23, 866-871. doi: 10.1016/j.copbio.2012.03.002

Tran, H. T., Hurley, B. A., and Plaxton, W. C. (2010a). Feeding hungry plants: the role of purple acid phosphatases in phosphate nutrition. Plant Sci. 179, 14-27. doi: 10.1016/j.plantsci.2010.04.005

Tran, H. T., Qian, W. Q., Hurley, B. A., She, Y. M., Wang, D. W., and Plaxton, W. C. (2010b). Biochemical and molecular characterization of AtPAP12 and AtPAP26: the predominant purple acid phosphatase isozymes secreted by phosphate-starved Arabidopsis thaliana. Plant Cell Envir. 33, 1789-1803. doi: $10.1111 /$ j.1365-3040.2010.02184.x
Vance, C. P., Uhde-Stone, C., and Allan, D. L. (2003). Phosphorus acquisition and use: critical adaptations by plants for securing a nonrenewable resource. New Phytol. 157, 423-447. doi: 10.1046/j.1469-8137.2003.00695.x

Veneklaas, E. J., Lambers, H., Bragg, J., Finnegan, P. M., Lovelock, C. E., Plaxton, W. C., et al. (2012). Opportunities for improving phosphorus-use efficiency in crop plants. New Phytol. 195, 306-320. doi: 10.1111/j.1469-8137.2012.04190.x

Venkidasamy, B., Selvaraj, D., and Ramalingam, S. (2019). Genome-wide analysis of purple acid phosphatase (PAP) family proteins in Jatropha curcas L. Int. J. Biol. Macromol. 123, 648-656.

Wang, L., Li, Z., Qian, W., Guo, W., Gao, X., Huang, L., et al. (2011). The Arabidopsis purple acid phosphatase AtPAP10 is predominantly associated with the root surface and plays an important role in plant tolerance to phosphate limitation. Plant Physiol. 157, 1283-1299. doi: 10.1104/pp.111. 183723

Wang, L., and Liu, D. (2018). Functions and regulation of phosphate starvationinduced secreted acid phosphatases in higher plants. Plant Sci. 271, 108-116. doi: 10.1016/j.plantsci.2018.03.013

Wang, L. S., Lu, S., Zhang, Y., Li, Z., Du, X., and Liu, D. (2014). Comparative genetic analysis of Arabidopsis purple acid phosphatases AtPAP10, AtPAP12, and AtPAP26 provides new insights into their roles in plant adaptation to phosphate deprivation. J. Integr. Plant Biol. 56, 299-314. doi: 10.1111/jipb.12184

Wang, X. R., Shen, J. B., and Liao, H. (2010). Acquisition or utilization, which is more critical for enhancing phosphorus efficiency in modern crops? Plant Sci. 179, 302-306. doi: 10.1016/j.plantsci.2010.06.007

Wu, W. W., Lin, Y., Liu, P. D., Chen, Q. Q., Tian, J., and Liang, C. Y. (2018). Association of extracellular dNTP utilization with a GmPAP1-like protein identified in cell wall proteomic analysis of soybean roots. J. Exp. Bot. 69, 603-617. doi: 10.1093/jxb/erx441

Xue, Y. B., Xiao, B. X., Zhu, S. N., Mo, X. H., Liang, C. Y., Tian, J., et al. (2017). GmPHR25, a GmPHR member upregulated by phosphate starvation, controls phosphate homeostasis in soybean. J. Exp. Bot. 68, 4951-4967. doi: 10.1093/jxb/ erx292

Xue, Y. B., Zhuang, Q. L., Zhu, S. N., Xiao, B. X., Liang, C. Y., Liao, H., et al. (2018). Genome wide transcriptome analysis reveals complex regulatory mechanisms underlying phosphate homeostasis in soybean nodules. Int. J. Mol. Sci. 19, 2924-2947. doi: 10.3390/ijms19102924

Yao, Z., Tian, J., and Liao, H. (2014). Comparative characterization of GmSPX members reveals that GmSPX3 is involved in phosphate homeostasis in soybean. Ann. Bot. 114, 477-488. doi: 10.1093/aob/mcu147

Yao, Z. F., Liang, C. Y., Zhang, Q., Chen, Z. J., Xiao, B. X., Tian, J., et al. (2014). PVSPX1 is an important component in the phosphorus signaling network of common bean regulating root growth and phosphorus homeostasis. J. Exp. Bot. 65, 3299-3310. doi: 10.1093/jxb/eru183

Yin, C. Y., Wang, F., Fan, H. Q., Fang, Y. M., and Li, W. F. (2019). Identification of tea plant purple acid phosphatase genes and their expression responses to excess iron. Int. J. Mol. Sci. 20:1954. doi: 10.3390/ijms20081954

Zhang, Q., Wang, C., Tian, J., Li, K., and Shou, H. (2010). Identification of rice purple acid phosphatases related to phosphate starvation signalling. Plant Biol. 13, 7-15. doi: 10.1111/j.1438-8677.2010.00346.x

Zhang, Z. L., Liao, H., and William, J. L. (2014). Molecular mechanisms underlying phosphate sensing, signaling, and adaptation in plants. J. Integr. Plant Biol. 56, 192-220. doi: 10.1111/jipb.12163

Zhao, J., Fu, J. B., Liao, H., He, Y., Nian, H., Hu, Y. M., et al. (2004). Characterization of root architecture in an applied core collection for phosphorus efficiency of soybean germplasm. Chinese Sci. Bull. 49, 1611-1620. doi: 10.1360/04wc0142

Zimmermann, P., Regierer, B., Kossmann, J., Frossard, E., Amrhein, N., and Bucher, M. (2004). Differential expression of three purple acid phosphatases from potato. Plant Biol. 6, 519-528. doi: 10.1055/s-2004-821091

Conflict of Interest: The authors declare that the research was conducted in the absence of any commercial or financial relationships that could be construed as a potential conflict of interest.

Copyright (C) 2020 Zhu, Chen, Liang, Xue, Lin and Tian. This is an open-access article distributed under the terms of the Creative Commons Attribution License (CC BY). The use, distribution or reproduction in other forums is permitted, provided the original author(s) and the copyright owner(s) are credited and that the original publication in this journal is cited, in accordance with accepted academic practice. No use, distribution or reproduction is permitted which does not comply with these terms. 